\title{
Type material of Phaonia (Diptera: Muscidae) in the Zoological Institute RAS, St-Petersburg, Russia
}

\section{Типовой материал Phaonia (Diptera: Muscidae) Зоологического института РАН, Санкт-Петербург, Россия}

\author{
Nikita E. Vikhrev", Oksana V. Vikhreva*, Olga G. Ovtshinnikova** \\ Никита Е. Вихрев ${ }^{*}$ Оксана В. Вихрева*, Омьга Г. Овчинникова*
}

\footnotetext{
* Zoological Museum of Moscow University, Bolshaya Nikitskaya 2, Moscow 125009, Russia. E-mail: nikita6510@ya.ru Зоологический музей, МГУ им. М.В. Ломоносова, Большая Никитская ул., 2, Москва 125009, Россия.

** Zoological Institute Russian Academy of Sciences, Universitetskaya naberezhnaya, 1, St-Petersburg 199034, Russia. Зоологический институт РАН, Университетская наб., 1, Санкт-Петербург 199034, Россия.
}

KEY WORDS: Diptera, Muscidae, Phaonia, Zoological Institute RAS, ZIN, type specimens.

КЛЮЧЕВЫЕ СЛОВА: Diptera, Muscidae, Phaonia, Зоологический институт РАН, ЗИН, типовой материал.

ABSTRACT. The type material of genus Phaonia in Zoological Institute RAS, St-Petersburg, Russia was revised and discussed. The data on other type specimens of the considered species of Phaonia were complied. This review is illustrated by photos of 44 types specimens of Phaonia kept in ZIN.

РЕЗЮМЕ. Изучены типовые экземпляры рода Phaonia, хранящиеся в Зоологическом институте РАН(Санкт-Петербург, Россия). Суммированы данные по местонахождению остальных типовых экземпляров рассмотренных видов Phaonia. Сделаны фотографии 44 типов Phaonia, хранящихся в Зоологическом институте РАН.

\section{Introduction}

In Zoological Institute, St-Petersburg, Russia (ZIN) rather rich type material of Muscidae is deposited. This material was properly examined by W. Hennig and subsequently published in his revision of the Palaearctice Muscidae. Since that, few more Muscidae were described from ZIN's material with one exception - the genus Phaonia Robineau-Desvoidy, 1830. In the period from 1980 till 1994 more than 30 taxa of Phaonia were described from the type material entirely or partly stored in ZIN. All relevant papers were published in Russian, most of these are not available on-line, the data were never reviewed.

We reexamined the type material of Phaonia in ZIN (totally 56 taxa) and found most of the type specimens to be in a good a condition. We also discuss where the rest parts of the type series are deposited or should be deposited according to the original descriptions and other literature data.

Our other task was finding of type localities which are given usually in Cyrillic alphabet and are from various remote corners of Russia, Soviet Central Asia, Mongolia and Central China. A lot of toponyms mentioned were renamed either in Soviet or post-Soviet periods. Especially difficult was to find localities for specimens collected by expeditions of the Russian Imperial Geographical Society in Central Asia under the leadership of P. Kozlov or V. Roborovsky. These localities were established according to the field reports published by Kozlov [1947] and Roborovsky [1949]. We did our best to bring all type localities to the level of geographical coordinates and present administrative divisions. In the lists of the type materials each label is first cited verbatim in the original language (usually Russian) and then followed by its English explanation, where the toponyms are mentioned in their modern form.

The paper is illustrated by 44 images of ZIN's types of Phaonia.

\section{Material and methods} lows:

Entomological collections are abbreviated as folBMNH — Natural History Museum, London, UK. MNM - Magyar Nemzeti Muzeum, Budapest, Hungary.

NRS - Naturhistoriska Riksmuseet, Stockholm, Sweden.

SDEI - Senckenberg Deutsches Entomologisches Institut, Müncheberg, Germany.

ZIN - Zoological Institute RAS, St-Petersburg, Russia.

ZMHU - Museum für Naturkunde, HumboldtUniversität zu Berlin, Germany.

ZMUH - Zoological Museum, Finnish Museum of Natural History, Helsinki, Finland.

ZMUM - Zoological Museum of Moscow University, Russia. 
Localities are given in the following form: country, region (for large countries), geographical coordinates, the later are given in the Decimal Degrees format.

\section{Type specimens of Phaonia in ZIN's col-} lection

\section{Phaonia adriani Zinovjev, 1994: 82 (Phaonia)} Fig. 1.

MATERIAL. ZIN: Holotype, O': Gergeti scree \& river 29503200 m USSR, Georgia Caucasus, Kazbegi, 11.VII.1983 A.C. Pon $\left(=\right.$ GEORGIA, $\left.\mathrm{H}^{\prime \prime} 42.66^{\circ} \mathrm{N} 44.58^{\circ} \mathrm{E}\right)$, Holotypus Phaonia adriani $\mathrm{O}^{\mathrm{T}}$ Zinovjev, 1994.

Other type material. BMNH: paratype, 19 , GEORGIA, the same label as the holotype, [Zinovjev, 1994].

\section{Phaonia alatavica Zinovjev, 1983: 183 (Phaonia)}

Fig. 21.

MATERIAL. ZIN: Holotype, Ơ: Алма-Ат. зап., 1650м устье Cp. Талгара Каспарян 6.VII.979 (= KAZAKHSTAN, Almaty reg., Alma-Atinsky Nat. Res, mouth Middle Talgar R., $43.225^{\circ} \mathrm{N}$ $77.290^{\circ} \mathrm{E}, 1650 \mathrm{~m}$ asl, D. Kasparian, 6.07.1979), Holotypus Phaonia alatavica $\mathrm{O}^{7}$ Zinovjev, 1982

Paratypes $30^{7} \sigma^{7}$, 3우: KAZAKHSTAN, Almaty reg.: the same label as the holotype, date 8.07.1979, $20^{7} \sigma^{2}$, 2оक; Алма-Ат. зап., 1800м Прав. Талгар, ельн. Каспарян (= Right Talgar R., fir forest, $43.210^{\circ} \mathrm{N} 77.283^{\circ} \mathrm{E}, 1800 \mathrm{~m}$ asl, D. Kasparian), 9.07.1979, $10^{7}, 1$ 우.

\section{Phaonia algida Zinovjev, 1983: 187 (Phaonia)} Fig. 2.

MATERIAL. ZIN: Holotype, ơ: Аксу-Дж.зап., 1600-1800м, ущ. Джабаглы Каспарян 3.VII.979 (= KAZAKHSTAN, S Kazakhstan reg., Aksu-Zhabagly Nat. Res., Zhabagly canyon, $42.4^{\circ} \mathrm{N}$ $70.5^{\circ} \mathrm{E}, 1600-1800 \mathrm{~m}$ asl, D. Kasparyan, 3.07.1979), Holotypus Phaonia algida $\mathrm{O}^{\top}$ Zinovjev, 1982

Phaonia alticiella Zinovjev, 1990: 491 (Phaonia)

MATERIAL. ZIN: paratypes, 2 +9 : MONGOLIA: Central aimak, $11 \mathrm{~km}$ OSO von Somon Bajanzogt $950 \mathrm{~m}$ Exp. Dr. Z. Kaszab (= Tov prov., $11 \mathrm{~km}$ SEE of Somon Bayantsogt, $48.05^{\circ} \mathrm{N} 105.98^{\circ} \mathrm{E}$ ), 26.07.1968, 19; Zavchan aimak am pass Chaldzan Sogotyn davaa, $2300 \mathrm{~m}$ Exp. Dr. Z. Kaszab (= Zavkhan prov., $48.6^{\circ} \mathrm{N} 97.2^{\circ} \mathrm{E}$ ), 14.07.1968, 1 .

Other type material. MNM [Zinovjev, 1990]. Holotype, ${ }^{\circ}$ : Uvs aimak, $2 \mathrm{~km} \mathrm{O}$ of Pass Ulaan davaa zw. See Orog nuur und Ulangom, 1950 m, Expedition of Dr. Kaszab 6.VII.1968 (= MONGOLIA, Uvs prov., $2 \mathrm{~km}$ E of Pass Ulaan davaa between Orog nuur Lake and Ulangom, $50.177^{\circ} \mathrm{N} 91.485^{\circ} \mathrm{E}, 6.07 .1968$ ).

Paratype 1+, MONGOLIA, Zavkhan prov., Zavchan aimak Choit chunch, $26 \mathrm{~km}$ ONO von See Telmen nuur, $2150 \mathrm{~m}$ Exp. Dr. Z. Kaszab (=26 km NEE of Telmen nuur Lake, $\left.48.7^{\circ} \mathrm{N} 97.8^{\circ} \mathrm{E}\right), 13$ 15.07.1968.

\section{Phaonia amurensis Hennig, 1963: 799 (Phaonia)} Fig. 3.

MATERIAL. ZIN: Holotype, Oج: Климоуцы Амур. обл. 40 км W Свободного Борисова 21. V. 959 дубово-лиственничн. лес на фоне черн. Березы (= RUSSIA, Amur reg., $40 \mathrm{~km} \mathrm{~W}$ of Svobodny, Klimoutsy, $51.46^{\circ} \mathrm{N} 127.59^{\circ} \mathrm{E}$, oak-larch forest with birch, K. Borisova, 27.07.1958), Phaonia amurensis Hennig n.sp. Holotypus.

Paratype, $10^{7}$, RUSSIA, the same label as the holotype.

Phaonia arida Zinovjev, 1983: 186 (Phaonia) Fig. 4.

MATERIAL. ZIN: Holotype, Ơ: Монголия, Баян Хонгорск аймак, ур. Дзун-Мод 70 км Ю Шинэ-Джинст Зайцев 1011.VIII.969 (= MONGOLIA, Bayankhongor prov., $70 \mathrm{~km} \mathrm{~S}$ of Shinejinst, Zuun Mod (= 100 trees, mongolian), $43.97^{\circ} \mathrm{N} 99.23^{\circ} \mathrm{E}$
$1300 \mathrm{~m}$ asl, V. Zaitsev, 10-11.08.1969), Holotypus Phaonia arida $\sigma^{T}$ Zinovjev, 1982

Paratypes, $20^{7} 0^{7}$, 1: IRAN, Sistan and Baluchestan reg., Хазыкъ верш Ку Туфтан Саргадъ Кирман (=Sarhad=Serhed plateau, Taftan Volcano, $28.6^{\circ} \mathrm{N} 61.1^{\circ} \mathrm{E}, \mathrm{N}$. Zarudny expedition to Persia), 21-26.08.1898, $10^{7}$;

KAZAKHSTAN, S Kazakhstan reg., Аксу-Джебаглинск. Зап. Южн. Казахст. обл. В. Шевченко 14-17.VI.953 (= Aksu-Zhabagly Nat. Res., $42.4^{\circ} \mathrm{N} 70.6^{\circ} \mathrm{E}, \mathrm{V}$. Shevchenko). $14-17.06 .1953,10^{\mathrm{T}}$;

TAJIKISTAN, Gorno-Badakhshan reg., Ванч, Дарваз Штакельберг (= Darvoz dist., Vanj vill., on Vanj River, $38.37^{\circ} \mathrm{N} 71.46^{\circ} \mathrm{E}$, $1750 \mathrm{~m}$ asl, A. Stackelberg), 30.07.1943, 1 ㅇ․

\section{Phaonia asiatica Hennig, 1963: 801 (Phaonia)}

Fig. 5.

MATERIAL. ZIN: Holotype, O’: Кондара, 1100 м д. Варзоза, Тадж. Гуссаковский 15.IX.945 (= TAJIKISTAN, Sughd reg., Varzob village, $38.79^{\circ} \mathrm{N} 68.82^{\circ} \mathrm{E}, 1100 \mathrm{~m}$ asl, V. Gussakovsky, 15.09.1945), Phaonia asiatica Hennig n.sp. Holotypus.

\section{Phaonia asierrans Zinovjev, 1981: 623 (Phaonia)}

Fig. 6.

MATERIAL. ZIN: Holotype, Ơ': Барабаш пойма Хасанский р-н Каспарян 2 VIII 978 (= RUSSIA, Primorsky reg., Khasansky distr., Barabashevka River flood plain, $43.19^{\circ} \mathrm{N} 131.50^{\circ} \mathrm{E}$, D. Kasparyan, 2.08.1978), Holotypus $\sigma^{7}$ Phaonia asierrans Zinovjev, 1981

Paratypes, $20^{7} 0^{7}, 1$ 1 : CHINA, Sichuan prov., Сычуань, Тацзынлу Потанин $\left(\right.$ Tachienlu=Dartsedo=Kangding, $30.05^{\circ} \mathrm{N}$ 101.96 ${ }^{\circ}$ E, G. Potanin), 6.06.1893, $10^{\top}$;

RUSSIA: Primorsky reg., Владивосток Седанка Зиновьев (= Vladivostok, Sedanka, 43.2€N 132.0€E, A. Zinovjev), 19.07.1979, 1; Sakhalin reg., о. Кунашир, окр. Южно-Курильска А. Зиновьев (= Kunashir Isl., Yuzhno-Kurilsk env., $44.03^{\circ} \mathrm{N} 145.86^{\circ} \mathrm{E}, \mathrm{A}$. Zinovjev), 3.07.1979, $10^{7}$.

Phaonia atrochaeta Zinovjev, 1980b: 909 (Phaonia) Fig. 7.

MATERIAL. ZIN: Holotype, о: Владивосток, Седанка A. Зиновьев 1.VI.1979 (= RUSSIA, Primorsky reg., Vladivostok, Sedanka, $43.2^{\circ} \mathrm{N} 132.0^{\circ} \mathrm{E}$, A. Zinovjev, 1.06.1979), Holotypus ${ }^{7}$ Phaonia atrochaeta Zinovjev, 1980

Phaonia chalchica Zinovjev, 1980a: 439 (Phaonia) Fig. 8 .

ZIN: Holotype, O': Холт, сев. Гоби Монголия Козлов 28.III12.V.926 (= MONGOLIA, Dundgovi prov., N Gobi, Khuld, $45.22^{\circ} \mathrm{N}$ 105.55E, H"1300 m asl, P. Kozlov), 28.03-12.05.1926.

Paratypes, $110^{7} 0^{7}$, 7우: MONGOLIA: the same label as the

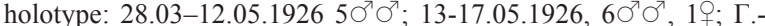
Алт. айм, Хасагт-Хайрхан 15 км Ю, Джаргалана, лов. Малеза, Зайцев Нарчук (= Govi-Altai prov., Hasagt Hayrhan Hills $15 \mathrm{~km} \mathrm{~S}$ of Jargalan, $46.85^{\circ} \mathrm{N} 95.90^{\circ} \mathrm{E}, \mathrm{H}^{\prime} 2000 \mathrm{~m}$ asl, V. Zaitsev, E. Narchuk) 14.08.1970 5+⿱ ; Центральный, аймак, Мунгэн Морьт, Зайцев (= Tov prov., Mongonmorit, $48.2^{\circ} \mathrm{N} 108.5^{\circ} \mathrm{E}, 1400 \mathrm{~m}$ asl, V. Zaitsev), 6-7.07.1967, 1우.

REMARKS. One male with the same label as the holotype was not found.

\section{Phaonia changaica Zinovjev, 1990: 500 (Phaonia)}

MATERIAL. ZIN: paratypes, $20^{7} \sigma^{7}$ : MONGOLIA: Bulgan Namnan ul Gebirge aimak, $3 \mathrm{~km}$ NW von Somon Chutag, $1150 \mathrm{~m}$ Exp. Dr. Z. Kaszab (= Bulgan prov., $3 \mathrm{~km} \mathrm{NW}$ of Khutag-Ondor sum, $49.42^{\circ} \mathrm{N} 102.66^{\circ} \mathrm{E}, 1000 \mathrm{~m}$ asl), $11.06-21.07 .1967,10^{7}$;

CHINA, Gansu prov., Горы Ганьсу, VIII 1871, 77460 Пржевальский (= Gansu Mts., H" $37^{\circ} \mathrm{N} 103^{\circ} \mathrm{E}$, Aug 1871, N. Przhevalsky), $10^{\circ}$. Other type material. MNM [Zinovjev, 1990]. Holotype, $0^{7}$, MONGOLIA, (Tov prov.) Central aimak $58 \mathrm{~km} \mathrm{NW}$ of Ulan-Baator $\left(=48.2^{\circ} \mathrm{N} 106.3^{\circ} \mathrm{E}\right), 1200 \mathrm{~m}$ asl, Exp. Dr .Z. Kaszab, 9.07.1964.

Paratypes, $40^{7} \sigma^{7}, 19$, MONGOLIA: (Tov prov.) Central aimak., $11 \mathrm{~km} \mathrm{SEE}$ of Bayantsogt, (H" 48.04 $\left.{ }^{\circ} \mathrm{N} 106.09^{\circ} \mathrm{E}\right), 1600 \mathrm{~m}$ asl, Exp. 


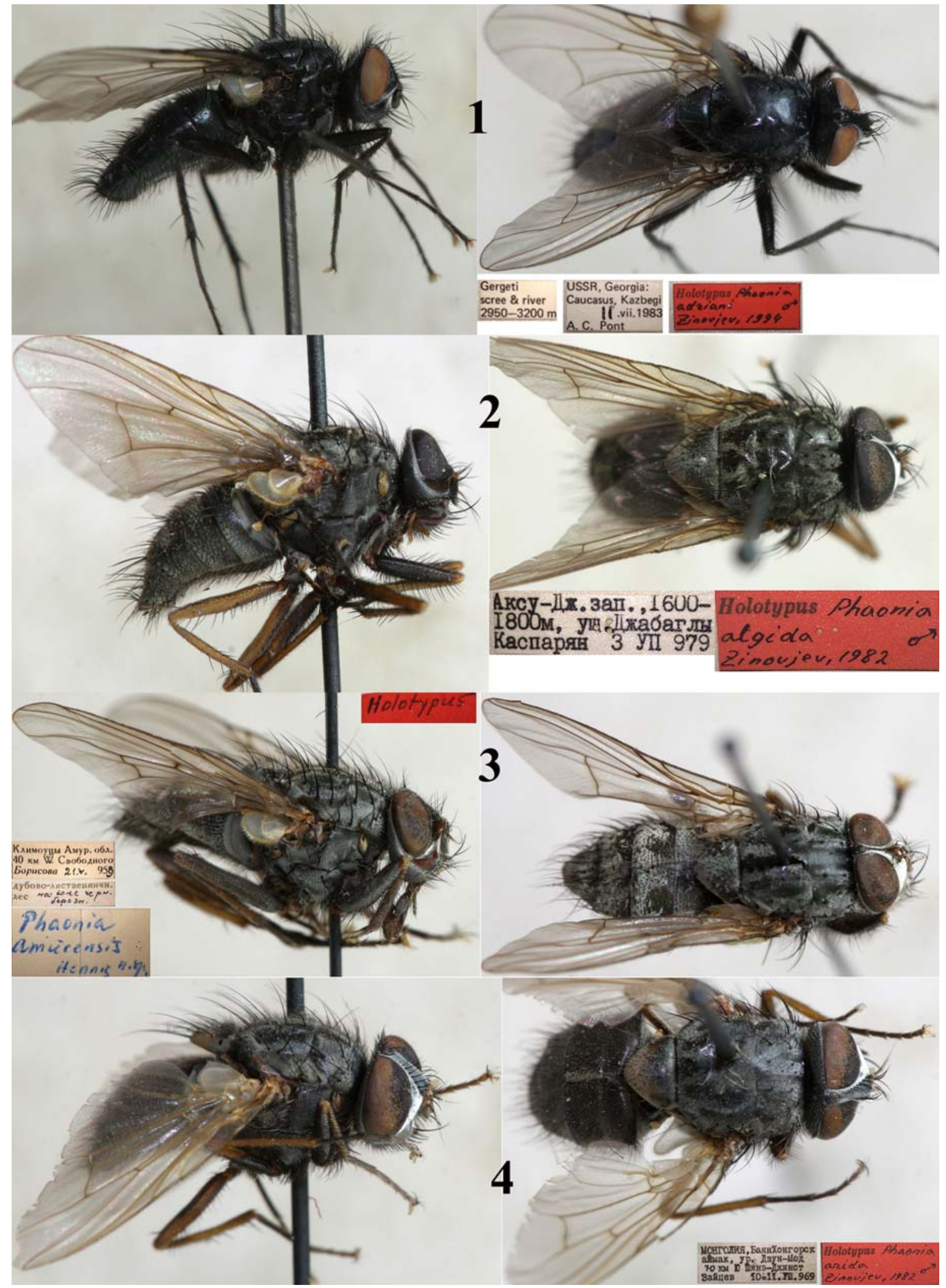

Figs 1-4. Phaonia spp., O' holotypes: 1 - P. adriani Zinovjev, 1994; 2 - P. algida Zinovjev, 1983; 3 - P. amurensis Hennig, 1963; $4-$ - P. arida Zinovjev, 1983.

Рис. 1-4. Phaonia spp., О голотипы: 1 - P. adriani Zinovjev, 1994; 2 - P. algida Zinovjev, 1983; 3 - P. amurensis Hennig, 1963 ; $4-$ - P. arida Zinovjev, 1983. 


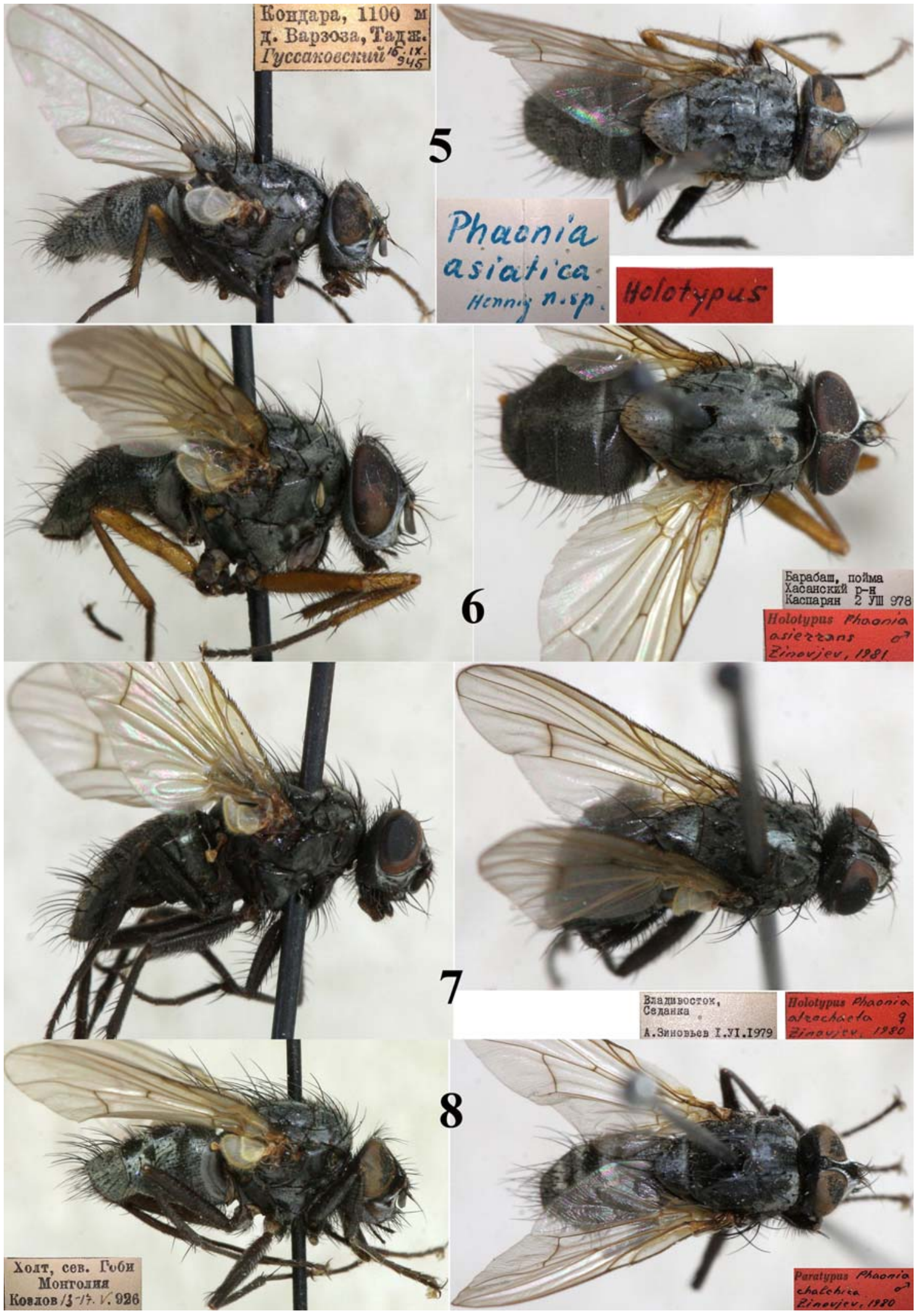

Figs 5-8. Phaonia spp. O' holotypes: 5 - P. asiatica Hennig, 1963; 6 - P. asierrans Zinovjev, 1981; 7 - P. atrochaeta Zinovjev, 1980; 8 - P. chalchica Zinovjev, 1980.

Рис. 5-8. Phaonia spp., О7 голотипы: 5 - P. asiatica Hennig, 1963; 6 - P. asierrans Zinovjev, 1981; 7 — P. atrochaeta Zinovjev, 1980; 8 - P. chalchica Zinovjev, 1980 
Dr .Z. Kaszab, 13.06-26.07.1968, 10; Arkhangai prov., Khangai mountains, $9 \mathrm{~km} \mathrm{NE}$ of Pass Egijn davaa (H"48 $\left.{ }^{\circ} \mathrm{N} 100^{\circ} \mathrm{E}\right), 2500 \mathrm{~m}$ asl, Exp. Dr .Z. Kaszab, 19.07.1966, $10^{7}$; Bulgan prov., 23 km NW of Khutag-Ondor sum $\left(49.52^{\circ} \mathrm{N} 102.44^{\circ} \mathrm{E}\right), 1150 \mathrm{~m}$ asl, Exp. Dr. Z

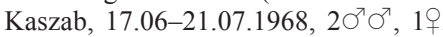

Phaonia cilitibia Emden, 1965: 261 (Phaonia)

MATERIAL. ZIN: Paratype, $10^{7}$ : (MYANMAR, Kachin st.), N. E. Burma Kambaiti $\left(25.53^{\circ} \mathrm{N} 98.14^{\circ} \mathrm{E}\right), 7000 \mathrm{ft} .=2130 \mathrm{~m}$ asl, R. Malaise, 11.05.1934, Phaonia cilitibia sp.n. paratype Van Emden det., 1953; Pres by Com.Inst.Ent., B.M. 1957-401.

Other type material. NRS: Holotype $\sigma^{7}$, the same locality as the above mentioned paratype, 7.05.1934.

NRS, BMNH and ZMUH: paratypes $300^{7} \sigma^{7}, 3$, 3 : the same locality, 6.04-7.06.1934 [Emden, 1965].

\section{Phaonia dahurica Zinovjev, 1990: 487 (Phaonia)}

MATERIAL. ZIN: type material was not found.

Other type material. MNM. Paratype, $1 \sigma^{7}$, MONGOLIA, Khovd prov., Bulgan aimak, $7 \mathrm{~km}$ NW von somon Chanzargalant, $1350 \mathrm{~m}$, (most probably «Bulgan aimak» is an error, if so the locality is: $7 \mathrm{~km}$ NW of Jargalant=Khovd, $\mathrm{H} \sim 48.03^{\circ} \mathrm{N} 91.60^{\circ} \mathrm{E}$ ), 16.06 .1968 Z. Kaszab. According to Zinovjev [1990], it should be in MNM.

\section{Phaonia decussata Stein, 1907: 321 (Aricia)} Fig. 9.

MATERIAL. ZIN: Lectotype, О’: исток Хуан хэ, с.-в. Тибетъ Козловъ 14.VI.900 (= CHINA, Qinghai prov., NE Tibet, source of Huang He River, Ngoring Lake or Gyaring Lake, $34.8^{\circ} \mathrm{N} 97.5^{\circ} \mathrm{E}$, $4270 \mathrm{~m}$ asl, P. Kozlov, 14.06.1900); Aricia decussata II.3 Stein (Stein's handwriting); Phaonia decussata Stein (Hennig's handwriting); Lectotypus Phaonia decussata Stein design. Zinovjev 1982 [Zinovjev, 1983].

Paralectotypes $2+0$, CHINA, the same label as the lectotype.

Other type material. $\mathrm{ZMHU}$, paralectotype,,+ , the same label as the lectotype [Pont, Werner, 2006].

Phaonia gergetica Zinovjev, 1994: 83 (Phaonia) Fig. 10.

MATERIAL. ZIN: Holotype, ơ: USSR, Georgia, Cuacasus, Kazbegi, Gergeti scree, 2950-3000 m, 14.VII.1983, A. C. Pont (= GEORGIA, Kazbegi, H" $42.66^{\circ} \mathrm{N} 44.58^{\circ} \mathrm{E}$ ), Holotypus Phaonia gergetica Zinovjev, 1994.

Paratypes, 10', 2 +q : GEORGIA, the same label as the holotype.

Other type material. BMNH [Zinovjev, 1994]. Paratypes $4 \sigma^{7} \sigma^{7}$ 4우: the same label as the holotype, dates 11-14.07.1983, 2950$3200 \mathrm{~m}$ asl, $4 \sigma^{\top} \sigma^{\top}, 3$ 우; the same label as the holotype, 10.07.1983, $2150 \mathrm{~m}$ asl, 1 ㅇ․

Phaonia gobertii sternalis Zinovjev, 1987: 440 (Phaonia)

Fig. 11.

MATERIAL. ZIN: Holotype, $\sigma^{7}$ : Владивосток Седанка A. Зиновьев 1.VI.1979 (= RUSSIA, Primorsky reg., Vladivostok, Sedanka, $43.2^{\circ} \mathrm{N} 132.0^{\circ} \mathrm{E}, 1.06 .1979$, A. Zinovjev), Holotypus Phaonia gobertii sternalis $\sigma^{7}$ Zinovjev ssp.n. 1985. Paratypes, $2 \sigma^{7} \sigma^{7}$, RUSSIA Primorsky reg.: Супутинский, Черемуха Н. Кр. (= Suputinsky $=$ Ussuriysky Nat. Res., $43.65^{\circ} \mathrm{N} 132.35^{\circ} \mathrm{E}$, from bird cherry tree, Prunus padus, N. Krivosheina), 18.04.1969, 107; Камень-Рыболов, Приморье, на заборе, А. Зиновьев (= Kamen-Rybolov, $44.73^{\circ} \mathrm{N}$ $132.04^{\circ} \mathrm{E}$, on fence, A. Zinovjev), 5.09.1978, $10^{7}$.

\section{Phaonia grunini Zinovjev, 1980a: 440 (Phaonia)} Fig. 12.

MATERIAL. ZIN: Holotype, $\sigma^{7}$ : р. Бугузун предгорье ю.-в. Алтай Грунин 29.VII.964 Holotypus $\sigma^{7}$ Phaonia grunini Zinovjev 1980 (= RUSSIA, Altai Republic. reg.,Buguzun R., H"50.0 N 89.1 ${ }^{\circ} \mathrm{E}$ H"1950 m asl, K. Grunin, 29.07.1964).

Paratypes $10^{7}, 1$ : RUSSIA, the same label as the holotype $10^{7}$; MONGOLIA, Южно-Гобийск айм. хр. Гурван-Сайхан 30 км BCB Байан-Далай Зайцев (= Omnogovi prov., $30 \mathrm{~km}$ NEE of
Bayandalai, $43.53^{\circ} \mathrm{N} 103.81^{\circ} \mathrm{E}, \mathrm{H}^{\prime} 2000 \mathrm{~m}$ asl, V. Zaitsev), $27-$ 28.08.1969, 1 우.

\section{Phaonia halophila Zinovjev, 1990: 479 (Phaonia)}

Fig. 13.

MATERIAL. ZIN: Holotype, $\sigma^{7}$ : Монголия, Кобдоский аймак, ур. Елхон 20 км ЮВ Алтая на Бодончи Нарчук 27. VII. 970 (= MONGOLIA, Khovd prov., $20 \mathrm{~km} \mathrm{~S}$ of (Mongolian) Altai Mts, on Bodonchiyn River, $45.88 \mathrm{~N}, 92.43^{\circ} \mathrm{E}$, E. Narchuk, 27.07.1970), Holotypus Phaonia halophila $\sigma^{7}$ Zinojev, 1988.

Paratypes $170^{7} \sigma^{7}, 12$ \% . MONGOLIA: the same label as the holotype, date 22-27.07.1970, collector V. Zaitsev or E. Narchuk, $60^{7} \sigma^{7}, 10$ ㅇ; Гоби-Алт, аймак, родн. Ушийн-Булак 30 км С3 Бэгэра, Нарчук (= Govi-Altai prov., $30 \mathrm{~km} \mathrm{NW}$ of Biger, $45.88^{\circ} \mathrm{N}$ $96.88^{\circ} \mathrm{E}, 1350 \mathrm{~m}$ asl, E. Narchuk), 13.07.1970, 3 ○ $^{\top}{ }^{\top}, 1$ 1 ; Гоби-Алт., аймак, родн. Хайчи-Була, 60 км ЮВ Бугата, Зайцев (= Govi-Altai prov., $60 \mathrm{~km} \mathrm{SE}$ of Bugat, $45.2^{\circ} \mathrm{N} 94.8^{\circ} \mathrm{E}$, V. Zaitsev), 19.07.1970, $2 \sigma^{7} \sigma^{7}$; ЮжноГобийск., аймак, оз. Баян-Тухум, 30км ЗСЗ Баян Далая, Зайцев (= Omnogovi prov., $30 \mathrm{~km}$ NWW of Bayandalai, Bayan-Tukhum L., $43.57^{\circ} \mathrm{N} 103.19^{\circ} \mathrm{E}$, V. Zaitsev), 31.07.1967, $20^{\top} \mathrm{O}^{\prime}$; Choved aimak, Jamatin Dolon cca $40 \mathrm{~km}, \mathrm{~N}$ von Somon Manchan am, SW Eske des char us nuur, 1200 m, Exp. Dr .Z. Kaszab, (= Khovd prov., $40 \mathrm{~km} \mathrm{~N}$ of Somon Mankhan=Tagrag, Khar us Nuur Lake shore, $47.75^{\circ} \mathrm{N} 92.05^{\circ} \mathrm{E}, 1200 \mathrm{~m}$ asl), $11-12.07 .1966,4 \sigma^{7} \sigma^{7}, 1$ ㅇ․

Other type material. MNM, paratypes, $260^{7} \sigma^{7}, 7+9 \circ$. MONGOLIA (all collected by Exp. Dr .Z. Kaszab): Khovd prov., $40 \mathrm{~km} \mathrm{~N}$ of Somon Mankhan=Tagrag, Char=Khar us Nuur Lake shore, $47.75^{\circ} \mathrm{N} 92.05^{\circ} \mathrm{E}, 1200 \mathrm{~m}$ asl), 11-12.07.1966, $19 \sigma^{\top} \sigma^{\top}, 3 \circ \circ$; Khovd prov., Chovd aimak, $3 \mathrm{~km} \mathrm{~N}$ von Somon Uench im Tal Uench gol, $1450 \mathrm{~m},\left(46.06^{\circ} \mathrm{N} 92.03^{\circ} \mathrm{E}\right), 2-3.07 .1966,2 \sigma^{7} \sigma^{7}, 3 \circ 0$; Uvs prov., am See Bag nuur, $6 \mathrm{~km}$ NO von Somon Zuungobi (Bag Nuur Lake, $6 \mathrm{~km}$ NE of Zuungovi, $\left.49.93^{\circ} \mathrm{N} 93.85^{\circ} \mathrm{E}\right), 1000 \mathrm{~m}, 25.06 .1968,10^{7}$; Tov prov., Central aimak, Tosgoni ovoo, 5-10 km N von UlanBaator, $1500-1700 \mathrm{~m},\left(48.02^{\circ} \mathrm{N} 106.95^{\circ} \mathrm{E}\right), 19-24.07 .1967,1 \mathrm{O}^{7}$; Govi-Altai prov., zwischen Beger nuur und Somon Beger=Biger $\left(45.71^{\circ} \mathrm{N} 97.18^{\circ} \mathrm{E}\right), 1400 \mathrm{~m}, 25-26.06 .1966,10^{7}$; Omnogovi prov., Tachilga ul, zw. Zogt-Ovoo=Tsogt Ovoo und Dalanzadgad $\left(44^{\circ} \mathrm{N}\right.$ $\left.105^{\circ} \mathrm{E}\right), 1550 \mathrm{~m}, 12.06 .1967,10^{7}$; Dornogovi prov., Ostgobi aimak, $40 \mathrm{~km}$ NW von Chara-Eireg (=Delgerekh? $\left.45.8^{\circ} \mathrm{N} 111.2^{\circ} \mathrm{E}\right), 1150$ $\mathrm{m}, 30.06 .1967,1 \mathrm{O}^{7}$; Bayankhongor prov., Oase Echin gol, $90 \mathrm{~km}$ NO von Grenzposten Caganbulag $(=90 \mathrm{~km} \mathrm{NE}$ of Caganbulag Checkpoint, $\left.\mathrm{H}^{\prime} 43.5^{\circ} \mathrm{N} 98.5^{\circ} \mathrm{E}\right), 950 \mathrm{~m}, 27-28.06 .1967,1$..

ZMUM: paratype, $10^{7}$, MONGOLIA, the same label as the holotype, but with V. Zaitsev as a collector.

\section{Phaonia hirtirostris Stein, 1907: 318 (Aricia)} Fig. 14.

MATERIAL. ZIN: Lectotype, O': в. б. оз. Орин-норъ бас. Хуан хэ 13900' Козлов. ку-нvi 01 (= CHINA, Qinghai prov., near Ngoring Lake, $34.8^{\circ} \mathrm{N} 97.5^{\circ} \mathrm{E}, 4270 \mathrm{~m}$ asl, P. Kozlov, late May - early June.1901) Lectotype $\sigma^{\top}$ Aricia hirtirostris Stein Des A. C. Pont 2000. Paralectotypes $80^{7} 0^{7}$ : CHINA, the same label as the holotype.

Other type material. ZMHU. Paralectotypes, $40^{7} 0^{7}$, CHINA, the same label as the lectotype [Pont, Werner, 2006].

Phaonia impura Zinovjev, 1987: 439 (Phaonia) Fig. 15.

MATERIAL. ZIN: Paratypes, $5 \sigma^{\top} \sigma^{\top}, 1+$. RUSSIA, Crimea reg., Sebastopol Tauria, V. Pliginski (=Sevastopol, V. Pliginski), $3 \sigma^{\top} \sigma^{\top}$ from pupae on the same single pin;

AZERBAIJAN, Nakhchivan reg., Нах АССР, Бузгов, на иве, М. Данилевский (= Ashagi-Buzgov, $39.51^{\circ} \mathrm{N} 45.40^{\circ} \mathrm{E}, 1400 \mathrm{~m}$ asl, on willow, M. Danilevsky), 14-17.04.1962, $2 \sigma^{\top} \sigma^{\top}, 1$,

Other type material. ZMHU. Holotype $\sigma^{7}$ and paratype 1 ; Prov. Sachsen Genthin Prof. P. Stein (= GERMANY, Saxony-Anhalt reg., Genthin, $52.40^{\circ} \mathrm{N} 12.15^{\circ} \mathrm{E}$ ), 7.06.1902 [Pont, Werner, 2006].

Phaonia irkutensis Zinovjev, 1990: 493 (Phaonia) Fig. 16.

MATERIAL. ZIN: Holotype, $\sigma^{7}$ : с. Молька, Балаг. p. Иркут. о. с ивы Рубцов 30.VI.931 (= RUSSIA, Irkutsk reg., 


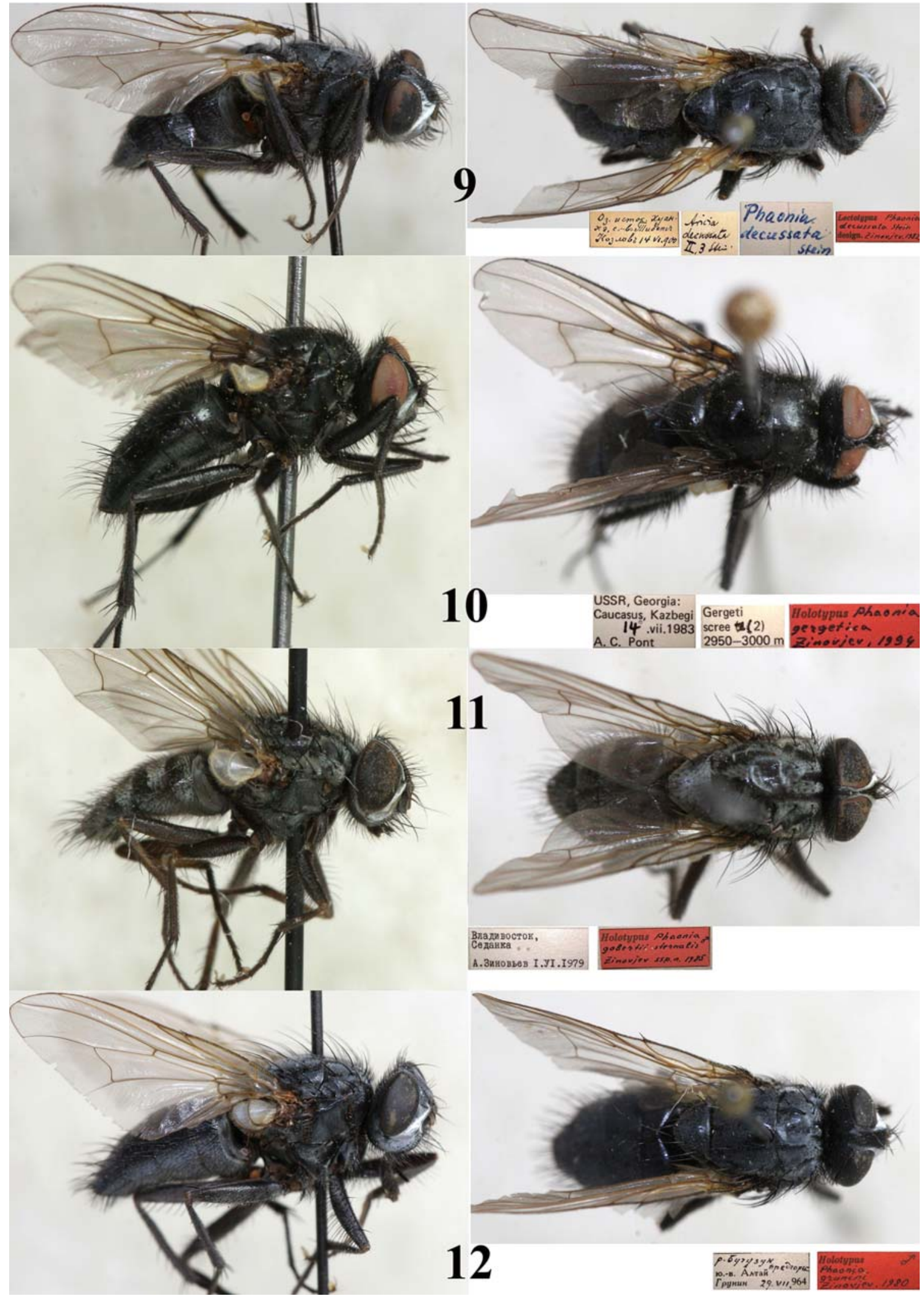

Figs 9-12. Phaonia spp., O' holotypes: $9-$ P. decussata Stein, 1907; $10-P$. gergetica Zinovjev, 1994; $11-$ P. gobertii sternalis Zinovjev, 1987; 12 - P. grunini Zinovjev, 1980,

Рис. 9-12. Phaonia spp., О' голотипы: 9 - P. decussata Stein, 1907; $10-P$. gergetica Zinovjev, 1994; 11 — P. gobertii sternalis Zinovjev, 1987; 12 - P. grunini Zinovjev, 1980. 


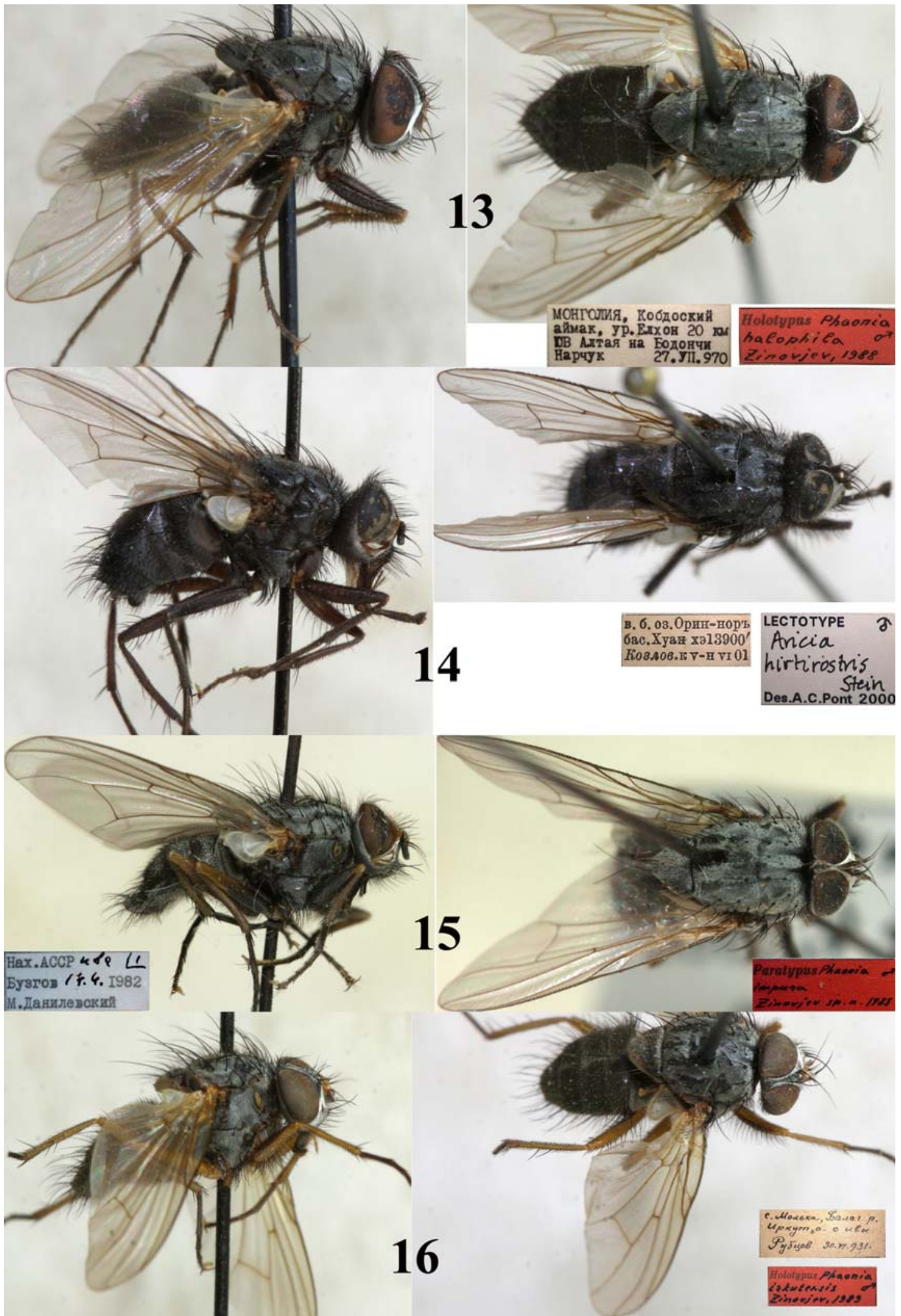

Figs 13-16. Phaonia spp.: 13 - P. halophila Zinovjev, 1990, $0^{\text {T }}$ holotype; $14-$ P. hirtirostris Stein, 1907, $O^{\text {T }}$ holotype; 15 - P. impura Zinovjev, 1987, O' paratype; $16-P$. irkutensis Zinovjev, 1990, $\sigma^{7}$ holotype.

Pис. 13-16. Phaonia spp.: 13 - P. halophila Zinovjev, 1990, О' голотип; 14 - P. hirtirostris Stein, 1907, О' голотип; 15 — $P$. impura Zinovjev, 1987, 1987, Oㄱ паратип; $16-P$. irkutensis Zinovjev, 1990, О7 голотип. 
Balagansky distr., Molka vill., $53.88^{\circ} \mathrm{N} 103.34^{\circ} \mathrm{E}$, from willow, I. Rubtsov, 30.06.1930), Holotypus $\sigma^{T}$ Phaonia irkutensis Zinovjev, 1989

Paratypes, 10 , 290. MONGOLIA: Селенгинск., аймак, 25 км В Дархана, разнотравно-караганов. Степь, Сугоняев (= Selenge prov., $25 \mathrm{~km}$ E of Dakhan, motley grass - Caragana steppe, E. Sugonyaev), 2.08.1975, 19; Убс. аймак,, 50 км ВЮВ Улангома, ловушка Малеза, Зайцев, Нарчук (= U⿻上丨 prov., 50 $\mathrm{km}$ SEE of Ulangom, $49.95^{\circ} \mathrm{N} 92.65^{\circ} \mathrm{E}$, Malaise trap, V. Zaitsev and E. Narchuk), 7.08.1975, 19; Bulgan aimak, Namnan u Gebirge, $5 \mathrm{~km}$ o von somon, Abzaga, $1400 \mathrm{~m}$, Exp. Dr. Z. Kascab (= Bulgan prov., Gurvanbulag-Avzaga, $47.75^{\circ} \mathrm{N} 103.40^{\circ} \mathrm{E}$ ), 2.07.1964, $10^{7}$

Other type material. MNM. Paratypes, $30^{7} \sigma^{7}, 2 \circ 9$, collected by Z. Kaszab [Zinovjev, 1990] should be in MNM: MONGOLIA Bayan-Olgii prov., im Tal des Flusses Chavcalyn gol, $25 \mathrm{~km}$ O von

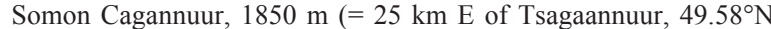
$\left.90.04^{\circ} \mathrm{E}\right), 3.08 .1968,10^{7}, 1$; ; Tov prov.: $11 \mathrm{~km}$ OSO von Somon Bajanzogt, $1600 \mathrm{~m}\left(11 \mathrm{~km}\right.$ SEE of Somon Bayantsogt, $48.05^{\circ} \mathrm{N}$ $\left.105.98^{\circ} \mathrm{E}\right), 26.07 .1968,10^{7}$; Tosgoni ovoo, $10 \mathrm{~km} \mathrm{~N}$ von UlanBaator, $1700-1900 \mathrm{~m}\left(48.02^{\circ} \mathrm{N} 106.95^{\circ} \mathrm{E}\right), 23-24.07 .1967,10^{7}$; Bayankhongor prov., Oase Echin gol, 90 km NO von Grenzposten Caganbulag (= $90 \mathrm{~km} \mathrm{NE}$ of Caganbulag Checkpoint, $\mathrm{H}^{\prime \prime} 43.5^{\circ} \mathrm{N}$ $\left.98.5^{\circ} \mathrm{E}\right), 950 \mathrm{~m}, 27-28.06 .1967,1$ ㅇ.

REMARKS. Several specimens are marked as "paratypes", but localities are not mentioned in the original description $50^{7} \mathrm{O}^{7}, 3$ 우 [Zinovjev, 1990]: Mongolia: Bulgan aimak, Namnan ul Gebirge, 23 $\mathrm{km}$ NW von Somon, Chutag $\left(49.51^{\circ} \mathrm{N} 102.49^{\circ} \mathrm{E}\right), 1150 \mathrm{~m}$ Exp. Dr Z. Kascab, 17.06.1968, , $10^{\prime}$; Хэнтэйский, аймак, пойма p. Онон, в окр Биндера или Баян-Адраги М. Козлов, И. Кержнер (Onon River flood-lands, from Binder, 48.65N, 110.60E to Bayan-Ardaga $48.57 \mathrm{~N}, 111.06^{\circ} \mathrm{E}$, M. Kozlov and I. Kerzhener), 4-7.07.1976, $20^{7} 0^{7}, 3$ 우; China, горы Ганьсу, VIII.1871, Пржевальский 77460 (= Gansu Mts., $\mathrm{H}^{\prime} 37^{\circ} \mathrm{N} 103^{\circ} \mathrm{E}$, Aug 1871, N. Przhevalsky), $10^{7}$; Былыра, Чит. обл., пойма реки Кыра, В. Рихтер (= Russia, Zabaykalsky reg., Bylyra village, flood-lands of Kyra River, $49.69^{\circ} \mathrm{N}$ $111.73^{\circ} \mathrm{E}, \mathrm{V}$. Rikhter), $19.06 .1975,10^{7}$.

Phaonia kambaitiana Emden, 1965: 236 (Phaonia)

MATERIAL. ZIN: Paratype, $10^{7}$ : (MYANMAR, Kachin st.), N. E. Burma Kambaiti $\left(25.53^{\circ} \mathrm{N} 98.14^{\circ} \mathrm{E}\right), 7000 \mathrm{ft}$, R. Malaise, 18.04.1934; Phaonia kambaitiana, sp.n. paratype, Van Emden det., 1953; Pres by, Com.Inst.Ent., B.M. 1957-401

Other type material. NRS: Holotype $\sigma^{7}$, the same locality, 7.05.1934.

NRS, BMNH and ZMUH: paratypes $280^{7} 0^{7}, 7$, 99 , the same locality as the above mentioned paratype [Emden, 1965].

Zoological Survey of India, Calcutta, paratypes: $10^{7}, 1$ 요, INDIA: (Uttarakhand st.), Dehradun $\left(30.3^{\circ} \mathrm{N} 78.0 \mathrm{E}\right)$ distr., $9000 \mathrm{ft} .=$ $2700 \mathrm{~m}$ asl, May.1910, 10'; (Himachal Pradesh st.), Shimla Hills, between Phagu and Kufri $\left(31.09^{\circ} \mathrm{N} 77.29^{\circ} \mathrm{E}\right), 8000-9000 \mathrm{ft} .=$ 2400-2700 m asl, 21.05.1916, 1우 [Emden, 1965].

\section{Phaonia kaszabi Zinovjev, 1990: 504 (Phaonia)}

MATERIAL. ZIN: paratypes, 2 +9 , MONGOLIA: Восточный аймак, Дучин-Гол 10 км СВ Гурван-Дзагала Нарчук (= Dornod prov., $10 \mathrm{~km} \mathrm{NE}$ of Gurvanzagal, $49.1^{\circ} \mathrm{N} 115.0^{\circ} \mathrm{E}, 800 \mathrm{~m}$ asl, E. Narchuk), 23.07.1975, 1우 Chovsgol aimak, 3 km SW von Somon Buren-chaan, 1650 m Exp. Dr. Z. Kaszab (= Khovsgol prov., 3 km SW of (Burentogtokh?, $\left.49.61^{\circ} \mathrm{N} 99.56^{\circ} \mathrm{E}\right), 16.07 .1968,1$ ㅇ.

Other type material. MNM. Holotype, $\sigma^{7}$ : Chentej aimak: zw. Somon Zenchermandal u. Somon Zargaltchaan, 1400 m, Exp. Dr .Z. Kaszab (MONGOLIA, Khentii prov., between Somon Tsenkhermandal and Somon Jargaltkhaan, $47.6^{\circ} \mathrm{N} 109.27^{\circ} \mathrm{E}, 1400 \mathrm{~m}$ asl), 27.07.1965.

Paratypes 3우. MONGOLIA, Khovsgol prov., Exp. Dr .Z. Kaszab: the same label as the holotype, 19; Chovsgol aimak, $4 \mathrm{~km}$ NW von der Stadt Moron, $1500 \mathrm{~m}$, Z. Kaszab (= $4 \mathrm{~km}$ NW of Moron, $\left.49.65^{\circ} \mathrm{N} 100.12^{\circ} \mathrm{E}\right), 19.07 .1968,1$; ; Chovsgol aimak, 3 $\mathrm{km} \mathrm{SW}$ von Somon Burenchaan, $1650 \mathrm{~m}(=3 \mathrm{~km}$ SW of (Burentogtokh (?) $\left.49.61^{\circ} \mathrm{N} 99.56^{\circ} \mathrm{E}\right), 21.06-16.07 .1968,1$ 우 [Zinovjev, 1990].
Phaonia kirgisorum Malianov, 1993: 421 (Phaonia) Fig. 17.

MATERIAL. ZIN: Holotype, $\sigma^{7}$ : Киргизский Алатау ущ.p. Мерке. 1500 на скале Малянов 19.VII.988 (= KAZAKHSTAN, Kyrgyz Alatau $=$ Kyrgyz Range, $20 \mathrm{~km} \mathrm{~S}$ of Merke, Merke R. gorge, $42.695^{\circ} \mathrm{N} 73.242^{\circ} \mathrm{E}$, on rock, M. Malyanov, 19.07.1988), Ph. kirgisorum Malianov sp.n. (голотип).

Phaonia kowarzii Schnabl, 1887: 837 (Aricia)

MATERIAL. ZIN: Syntype, O': Aricia kowarzii Schn. Mohylev-Gouv. Syntype $\sigma^{T}$ Aricia kowarzii Schnabl Conf. A. C. Pont 2000 (= BELARUS, Mogilev reg.).

\section{Phaonia liliputa Zinovjev, 1990: 498 (Phaonia)}

MATERIAL. ZIN: paratypes, $50^{\top} \sigma^{7}$ : Central aimak, SO von Somon Bajanzogt $1600 \mathrm{~m}$ Exp. Dr. Z. Kaszab, (= MONGOLIA, Tov (Central) prov., SE of Bayantsogt, $\mathrm{H}^{\prime} 47.88^{\circ} \mathrm{N} 106.29^{\circ} \mathrm{E}$ ), 11.06.1966.

Other type material. MNM: Holotype $\sigma^{7}$ and the rest of paratypes. According to Zinovjev [1990] in MNM should be $160^{7} 0^{7}$, 8 우 paratypes: MONGOLIA: the same label as ZIN's paratypes,

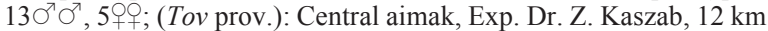
$\mathrm{SE}$ von Ulan-Baator, Nucht im Bogdo $\left(47.84^{\circ} \mathrm{N} 107.16^{\circ} \mathrm{E}\right)$, 6.07.1964, 2우; Tosgoni ovoo, 6-10 km N von Ulan-Baator, 1700 m, 7-8.06.1964, 1 ; 23-24.07.1967, $20^{7} \mathrm{O}^{7}$; Changaj Gebirge, $8 \mathrm{~km}$ $\mathrm{W}$ von somon Urdtamir, $1620 \mathrm{~m}$, Z. Kaszab) (= Arkhangai prov., Khangai Mts, somon Ikh Tamir, Urd Tamir gol $=\mathrm{S}$ Tamir River, $\left.47.5^{\circ} \mathrm{N} 101.0^{\circ} \mathrm{E}\right), 22.07 .1966,10^{\top}$.

\section{Phaonia longipalpis Emden, 1965: 264 (Phaonia)}

MATERIAL. ZIN: Paratypes, $20^{\top} 0^{7}$ : (MYANMAR, Kachin st.), N. E. Burma Kambaiti $\left(25.53^{\circ} \mathrm{N} 98.14^{\circ} \mathrm{E}\right), 2000 \mathrm{~m}$, R. Malaise, 4.06.1934; Phaonia longipalpis sp.n. paratype, Van Emden det., 1953; Pres by, Com.Inst.Ent., B.M. 1957-401.

Other type material. NRS: Holotype $\sigma^{7}$, the same locality as the above mentioned paratype, 1.06 .1934 .

NRS, BMNH and ZMUH: paratypes, $730^{7} \sigma^{7}, 28$ 우, the same locality, 6.04-17.06.1934 [Emden, 1965].

\section{Phaonia lutescens Zinovjev, 1990: 482 (Phaonia)} Fig. 42.

MATERIAL. ZIN: paratypes, $20^{7} \sigma^{\top}, 1$ : 1 : MONGOLIA, Bajanchongoraimak, Oase Echin gol, $90 \mathrm{~km}$ NO von Grenzposten Caganbulag, $950 \mathrm{~m}$, Exp. Dr. Z. Kaszab (= Bajanchongor prov., $90 \mathrm{~km} \mathrm{NE}$ of Caganbulag Checkpoint, $\left.\mathrm{H}^{\prime \prime} 43.5^{\circ} \mathrm{N} 98.5^{\circ} \mathrm{E}\right), 27-29.06 .1967$.

Other type material. MNM. Holotype, $O^{7}$ : MONGOLIA, Bajanchongoraimak, Oase Echin gol, $90 \mathrm{~km}$ NO von Grenzposten Caganbulag, $950 \mathrm{~m}$ Exp. Dr. Z. Kaszab (= Bajanchongor aimak, 90 $\mathrm{km}$ NE of Caganbulag Checkpoint, $\left.\mathrm{H}^{\prime \prime} 43.5^{\circ} \mathrm{N} 98.5^{\circ} \mathrm{E}\right), 27-$ 29.06.1967.

Paratypes: $40^{7} 0^{7}$, 4 우: MONGOLIA: the same label as the holotype, $4 \bigcirc^{7} \sigma^{7}$, 290; Central aimak, Tosgoni ovoo, 5-10 km N von Ulan-Baator, 1500-1700 m, 19-20 and 23-24.VII.1967, Exp. Dr. Z. Kaszab (= Tov prov., $5-10 \mathrm{~km} \mathrm{~N}$ of Ulan-Baator, $48.0^{\circ} \mathrm{N} 106.9^{\circ} \mathrm{E}$ ), 19-24.07.1967, 2우.

Phaonia malaiseana Emden, 1965: 257 (Phaonia)

MATERIAL. ZIN: Paratype, 10': (MYANMAR, Kachin st.), N. E. Burma Kambaiti $\left(25.53^{\circ} \mathrm{N} 98.14^{\circ} \mathrm{E}\right), 2000 \mathrm{~m}$, R. Malaise, 18.04.1934; Phaonia longipalpis sp.n. paratype, Van Emden det., 1953; Pres by, Com.Inst.Ent., B.M. 1957i401. Other type material. NRS: Holotype $\sigma^{7}$, the same locality as the above mentioned paratype, 7.05.1934. NRS, BMNH and ZMUH: patatypes, $280^{\top} 0^{\top}$, 7우, the same locality, 6.04-4.06.1934 [Emden, 1965].

Phaonia marakandensis Hennig, 1963: 843 (Phaonia)

Fig. 22.

MATERIAL. ZIN: Holotype, O': ствол дерева Самарканд Узб. Сычевская 21.IV.1961 (= UZBEKISTAN, Samarkand, $39.6^{\circ} \mathrm{N}$ 


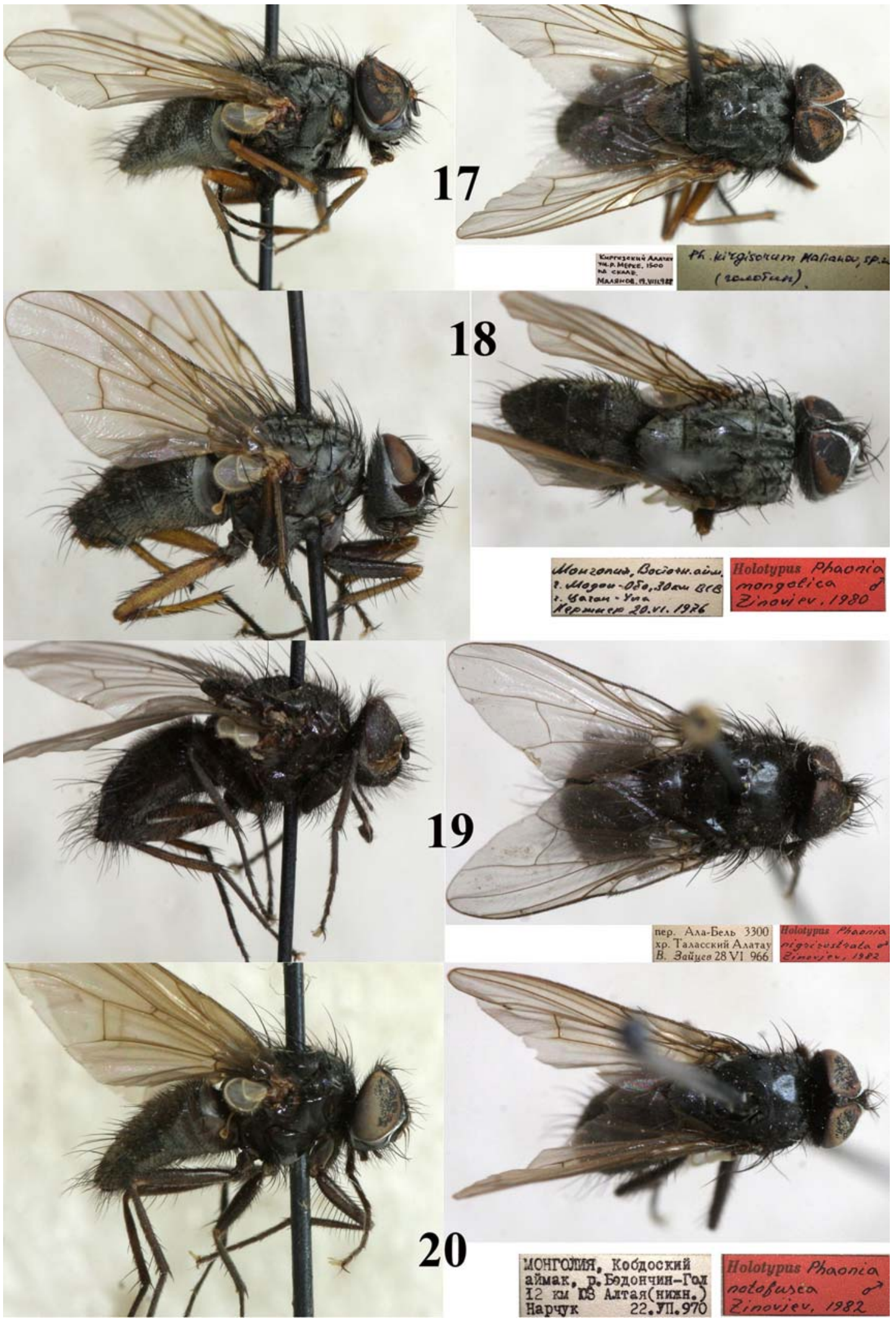

Figs 17-20. Phaonia spp., O $^{7}$ holotypes: 17 - P. kirgisorum Malianov, 1993; 18 - P. mongolica Zinovjev, 1980; 19 - P. nigrirostrata Zinovjev, 1983; $20-P$. notofusca Zinovjev, 1983.

Рис. 17-20. Phaonia spp., O’ голотипы: 17 - P. kirgisorum Malianov, 1993; 18 - P. mongolica Zinovjev, 1980; 19 — P. nigrirostrata Zinovjev, 1983; $20-P$. notofusca Zinovjev, 1983. 
$67.0^{\circ} \mathrm{E}, \mathrm{V}$. Sychevskaya, 21.04.1961), Holotypus Phaonia marakandensis n.sp. Hennig.

\section{Phaonia modesta Sorokina, 2015: 579 (Phaonia)}

MATERIAL. ZIN: Holotype, ơ, UZBEKISTAN, Samarqand reg., Samarkand, H'39.6 ${ }^{\circ} \mathrm{N} 67.0^{\circ} \mathrm{E}, \mathrm{V}$. Sychevskaya, emergence from faeces 8.05.1949.

Paratypes $110^{7} \sigma^{\top}, 7+9$. UZBEKISTAN, Samarqand reg.: the same label as the holotype, $10^{7}$; the same label as the holotype, but date 15.04.1949, $4 \sigma^{7} \sigma^{7}$; the same label as the holotype, but emergence from dump and date 26.04.1949, $10^{7}, 2$, $9+$; the same label as the holotype, but date 31.03.1949, $2 \sigma^{\top} \sigma^{7}, 3$ o+ ; Fergana reg.: Shakhimardan, $39.98^{\circ} \mathrm{N} 71.80^{\circ} \mathrm{E}$ (actually Shakhimardan is an exclave of Uzbekistan, surrounded by Kyrgyzstan), V. Sychevskaya, 29.08.1955, $10^{7}$; Fergana, $40.4^{\circ} \mathrm{N} 71.8^{\circ} \mathrm{E}, 8.05 .1953$, V. Sychevskaya, $10^{7}$; Surxondaryo reg.: Termez, $37.2^{\circ} \mathrm{N} 67.3^{\circ} \mathrm{E}$, V. Sychevskaya, 24.02.1958, $10^{7}$; Baysun $38.2^{\circ} \mathrm{N} 67.2^{\circ} \mathrm{E}$, V. Sychevskaya 9.06.1957, 1 우

TURKMENISTAN, Balkan reg., Kara-Kala, $38.43^{\circ} \mathrm{N} 56.30^{\circ} \mathrm{E}$ bank of Sumbar River, Ushinsky, 1933, 19 (modified from [Sorokina, 2015])

Other type material. ZMUM, paratypes, $2 \sigma^{7} \sigma^{7}$ : TURKMENISTAN, Ahal reg., Geok Tepe env., Chuli River gorge in Kopet Dag Range, $38.0^{\circ} \mathrm{N} 58.0^{\circ} \mathrm{E}, \mathrm{N}$. Krivosheina, emergence from fungus 10 $13.06 .1984,10^{7}$; TAJIKISTAN, Sughd reg., Varzob village, $38.79^{\circ} \mathrm{N}$ $68.82^{\circ} \mathrm{E}, 1100 \mathrm{~m}$ asl, A. Romanov, 14.05.1939, $10^{7}$.

\section{Phaonia mongolica Zinovjev, 1980: 442 (Phaonia)} Fig. 18.

MATERIAL. ZIN: Holotype, $\sigma^{7}$ : Монголия, Восточн. айм. г. Модон-Обо, 30км ВСВ х. Цаган-Ула Кержнер 20.VI.1976 (= MONGOLIA, Dornod prov., Modon-Obo Mt, $30 \mathrm{~km} \mathrm{NEE} \mathrm{of} \mathrm{Tsa-}$ gan-Ula Range, $46.9^{\circ} \mathrm{N} 117.0^{\circ} \mathrm{E}$, I. Kerzhener), 20.06.1976. Holotypus Phaonia mongolica $\sigma^{\top}$ Zinovjev, 1980.

Paratypes, $1 \sigma^{7}, 1+$. MONGOLIA: the same label as the holotype, 1 웅 Восточный, аймак, г. Дерхан-Цаган-, Обо, 60 км ВСВ БаянБурда, Кержнер (= Dornod prov., Derkhan-Tsagaan-Ovoo Mt., $46.8^{\circ} \mathrm{N} 118.2^{\circ} \mathrm{E}$, I. Kerzhener), 2-4.08.1976, $10^{7}$.

Phaonia nigrirostrata Zinovjev, 1983: 190 (Phaonia) Fig. 19.

MATERIAL. ZIN: Holotype, о: пер. Ала-Бель 3300 хр. Таласский Алатау В. Зайцев 28.VI.966 (= KYRGYZSTAN, Talas Alatau Range, Alabel Pass, $42.25^{\circ} \mathrm{N} 73.05^{\circ} \mathrm{E}, 3300 \mathrm{~m}$ asl, V. Zaitsev, 28.06.1966), Holotypus Phaonia nigrirostrata $O^{7}$ Zinovjev, 1982.

Paratypes $12 \sigma^{7} \sigma^{7}, 16$ 우. KYRGYZSTAN: the same label as the holotype, 1; Терскей Алатоо, Чон Кызыл-су, Второв (= Terskey Alatau Range, Chong Kyzyl-Suu valley, $42.28^{\circ} \mathrm{N} 78.11^{\circ} \mathrm{E}, \mathrm{P}$. Vtorov)

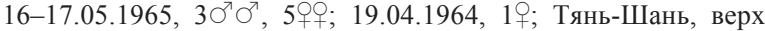
реки Б. Нарын, 3500 м, Злотин (= Tian-Shan, upper reaches of B Naryn River, $3500 \mathrm{~m}$ asl, $41.73^{\circ} \mathrm{N} 77.89^{\circ} \mathrm{E}$, R. Zlotin), 9.07.1965, $1 \sigma^{\top}, 1+1$; $12.06 .1965,1 \sigma^{\top} ; 27.06 .1965,1 \sigma^{\top} ; 1.07 .1965,1 \sigma^{\top}$ $3.07 .19652 \sigma^{7} \sigma^{7} ; 24.07 .1964,1 \sigma^{7}$; ледн. Кара-Баткак, 3300 м, Терскей Алатоо, Второв (= Terskey Alatau, Kara-Batkak glacier, $3300 \mathrm{~m}$ asl, $42.17^{\circ} \mathrm{N} 78.26^{\circ} \mathrm{E}, \mathrm{P}$. Vtorov), 31.05.1965, $10^{7}$; ТяньШань, p. Куйлю, П. Второв (= Tian Shan, Kuylu R., H" $42.4^{\circ} \mathrm{N}$ $78.5^{\circ}$ E, P. Vtorov), 4.07.1962, $10^{7}$; пер. Тюшасу, 3200, хр. Кирг. Алатау, В. Зайцев (= Kyrgyz Ala-Too Range, Tuu-Ashu or TyuzAshu Pass, 3200 m asl, V. Zaitsev) 30.05.1966, 19; Чайсандк Мерке, Ауліэат. у. (9450), Гольбек (= Sandyk Range, Merke River valley, $9450 \mathrm{ft} .=2880 \mathrm{~m}$ asl, $42.54^{\circ} \mathrm{N} 73.33^{\circ} \mathrm{E}$, A. Golbek) 22.06.1910, 1; Терскей Алатау, ю. Каракола, Кашкаров (= Terskey Alatau, S of Karakol, H" $42.3^{\circ} \mathrm{N} 78.5^{\circ} \mathrm{E}$, D. Kashkarov) $17-$ 22.07.1934, 3 90; 26.07.1934, 19; у ледн. Совнаркома, ист. Нарына Семир, Кашкаров (= Semirechye or Zhetysu, source of Naryn River, $41.77^{\circ} \mathrm{N} 77.97^{\circ} \mathrm{E}$, D. Kashkarov), 23.07.1934, 1 ; ; Нарынтау. 30 км О г. Нарын, верш. горы субальпийск. луг К. Городков (= $30 \mathrm{~km}$ E of Naryn town, mountain peak, subalpine meadow, H"41.45 $\mathrm{N} 76.41^{\circ} \mathrm{E}, \mathrm{K}$. Gorodkov), 8.08.1969, 1 웅.

Other type material. ZMUM:.paratype, 19 , хр. Терскей, Чонкызылсу, Станция АН, Д. Панфилов (= KYRGYZSTAN, Terskey
Alatau Range, Chong Kyzyl-Suu valley, research station, $42.28^{\circ} \mathrm{N}$ $78.11^{\circ}$ E, D. Panfilov), 24.06.1953, with A. Pont's 1971 label Phaonia hirtirostris and Zinovjev's label Phaonia nigrirostrata Zinovjev, 1982, paratypus.

REMARKS. Four specimens listed as paratypes by $\mathrm{Zi}$ novjev [1983] were not found, possibly they are in BMNH.

Phaonia notofusca Zinovjev, 1983: 184 (Phaonia) Fig. 20.

MATERIAL. ZIN: Holotype, $\sigma^{7}$ : Монголия, Кобдоский аймак, р. Бодончин-Гол 12 км ЮЗ Алтая (нижн.) Нарчук 22.VII.970 (= MONGOLIA, Khovd prov., $12 \mathrm{~km} \mathrm{SW}$ of Mongolia Altai Mts, on Bodonchiyn River, 45.94N, 92.50 E, E. Narchuk, 22.07.1970), Holotypus Phaonia notofusca Zinovjev, 1982.

Paratypes $290^{7} \sigma^{7}, 609$. MONGOLIA: the same label as the holotype, collector E. Narchuk or V. Zaitsev, 22-27.07.1970, $21 \sigma^{7} \sigma^{7}$, 6 कо; Кобдоский аймак, родн. Нарийн-Булак, хр.Их-ХавтгийнНуру, Нарчук (= Khovd prov., Nariyn-Bulak water sorce, H"45.94N, $92.50^{\circ}$ E, E. Narchuk), 24.07.1970, $3 \sigma^{\top} \sigma^{7}$; Гоби-Алт. Аймак, родн. Хайчи-Булак, 60 км ЮВ Бугата, Нарчук (= Govi-Altai prov.,60 $\mathrm{km}$ SE of Bugat, $45.2^{\circ} \mathrm{N} 94.8^{\circ} \mathrm{E}$, E. Nurchuk), 19.07.1970, $3 \mathrm{O}^{\top} \mathrm{O}^{\top}$; KYRGYZSTAN, p. Чу 10 км южн. Рыбачье Кирг. 1600 м, Нарчук $\left(=10 \mathrm{~km} \mathrm{~S}\right.$ of Balykchy=Rybachie, Chu River, $42.39^{\circ} \mathrm{N} 76.09^{\circ} \mathrm{E}$, $1600 \mathrm{~m}$ asl, E. Narchuk), 5.08.1969, $2 \sigma^{7} \sigma^{7}$

Other type material. ZMUM: paratype, MONGOLIA, $10^{7}$, the same label as the holotype.

One paratype mentioned in the description [Zinovjev, 1983], Кобдоский аймак, ур. Елхон 20 км ЮВ Алтая на Бодончи Нарчук (= MONGOLIA, Khovd prov., $20 \mathrm{~km} \mathrm{~S}$ of (Mongolian) Altai Mts, on Bodonchiyn River, $45.88^{\circ} \mathrm{N} 92.43^{\circ} \mathrm{E}$, E. Narchuk), 27.07.1970, $10^{7}$, was not found.

\section{Phaonia nudiseta Stein, 1907: 318 (Aricia)} Fig. 41

MATERIAL. ZIN: Lectotype, $\sigma^{7}$ : p. Кундур чю 135т' Камъ, бас. Голубой Козлов 12 v 01 (= CHINA, Sichuan prov., Yangtze River basin, Kundur chu R., H'33.2 ${ }^{\circ} \mathrm{N} 98.1^{\circ} \mathrm{E}, 4100 \mathrm{~m}$ asl, 12.05.1901, P. Kozlov (see discussion on type locality of Lispe brunnicosa [Vikhrev, 2012: 431]), Lectotype $\sigma^{7}$ Aricia nudiseta Stein Des A. C. Pont 2000 .

Paralectotypes $2 \sigma^{7} \sigma^{7}$, CHINA, the same label as the holotype.

Other type material. ZMHU, paralectotype, $1 \sigma^{7}$, CHINA, the same label as the lectotype (but the collector is A. Kaznakov and the altitude is $4050 \mathrm{~m}$ asl) is in ZMHU [Pont, Werner, 2006].

Phaonia obsoleta Hennig, 1963: 849 (Phaonia) Fig. 43.

MATERIAL. ZIN: Holotype, О7: пер. Анзоб. Гиссар. хр., Тадж Грунин 30.VI.956 (= TAJIKISTAN, Sughd reg., Gissar Range, Anzob pass, $39.08^{\circ} \mathrm{N} 68.87^{\circ} \mathrm{E}, \mathrm{H} " 3300 \mathrm{~m}$ asl, 30.06.1956, K. Grunin), Phaonia obsoleta n.sp. Hennig Holotypus.

\section{Phaonia ommatina Zinovjev, 1981: 624 (Phaonia)} Fig. 23.

MATERIAL. ZIN: Holotype, O?: о. Кунашир, окр. ЮжноКурильска, 9км влк. Менделеева Зиновьев 9.VII.1979 (= RUSSIA, Sakhalin reg., Kunashir Isl., 9 km from Yuzhno-Kurilsk, Mendeleev volcano, $43.98^{\circ} \mathrm{N} 145.75^{\circ} \mathrm{E}$, A. Zinovjev, 9.07.1979), Holotypus Phaonia ommatina $O^{T}$ Zinovjev, 1981.

Paratypes, $14 \sigma^{7} O^{7}, 1$, , RUSSIA, Sakhalin reg.: the same label as the holotype, $10^{\text {T}}$; о. Кунашир, окр., Южно-Курильска, Зиновьев (= Kunashir Isl., Yuzhno-Kurilsk $\left(44.03^{\circ} \mathrm{N} 145.86^{\circ} \mathrm{E}\right)$ env., A. Zinovjev), 4-7.07.1979, $120^{7} \sigma^{7}, 1$; ; Курильск, о. Итуруп, В. Рихтер 25.VI.968 (= Iturup Isl., Kurilsk, $45.25^{\circ} \mathrm{N} 147.89^{\circ} \mathrm{E}, \mathrm{V}$. Rikhter), 25.06.1968, $10^{7}$.

Other type material. ZMUM: paratype, $10^{7}$ : о. Кунашир, окр., Южно-Курильска, Зиновьев (= RUSSIA, Sakhalin reg., Kunashir Isl., Yuzhno-Kurilsk $\left(44.03^{\circ} \mathrm{N} 145.86^{\circ} \mathrm{E}\right)$ env., A. Zinovjev), 5.07.1979.

One paratype mentioned in the description [Zinovjev, 1981] (the same label as the holotype, $10^{7}$ ) was not found. 


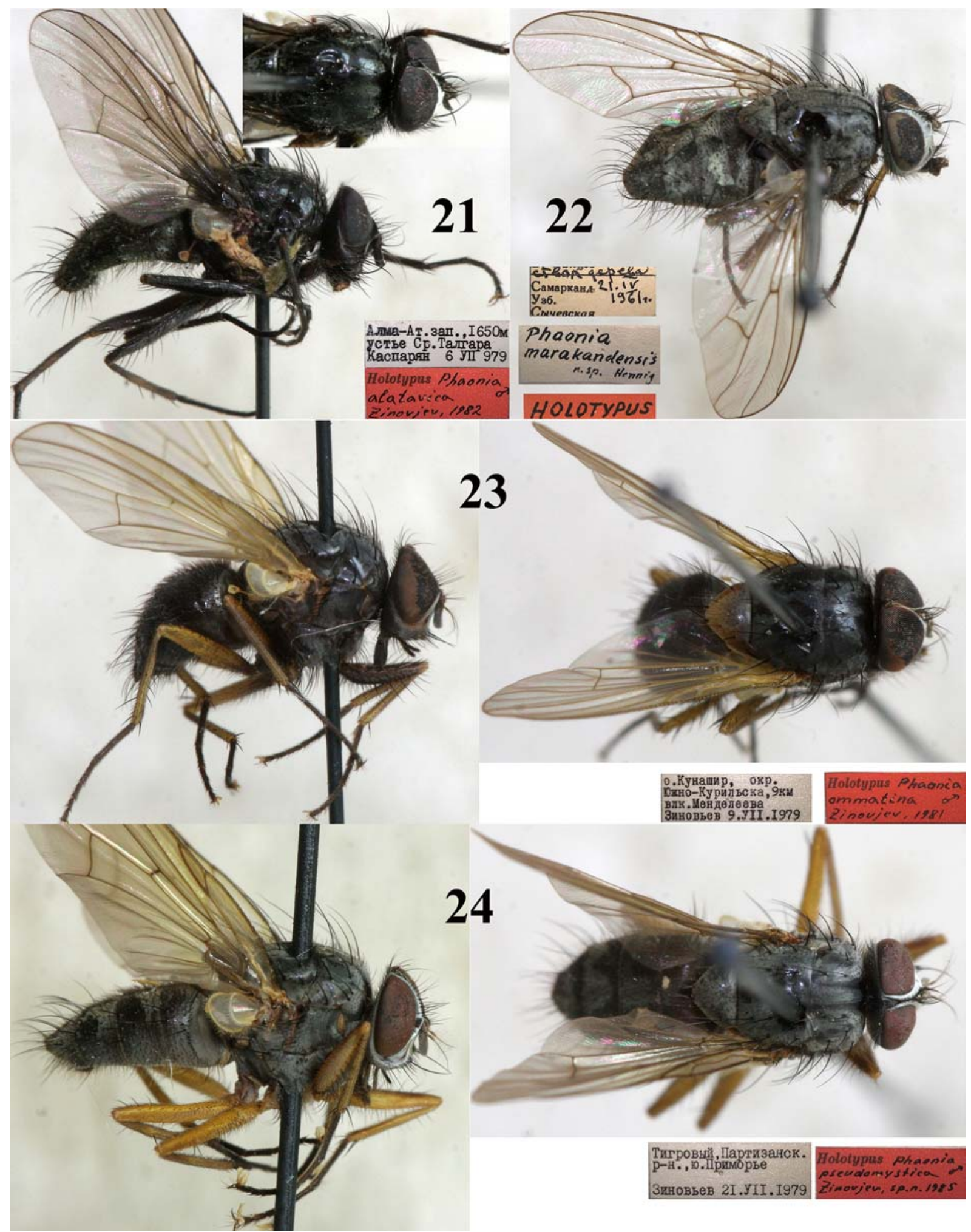

Figs 21-24. Phaonia spp., OT holotypes: $21-$ P. alatavica Zinovjev, 1983; $22-$ P. marakandensis Hennig, 1963; 23 - P. ommatina Zinovjev, 1981; 24 - P. pseudomystica Zinovjev, 1987.

Рис. 21-24. Phaonia spp., О7 голотипы: 21 - P. alatavica Zinovjev, 1983; 22 - P. marakandensis Hennig, 1963; 23 - P. ommatina Zinovjev, 1981; 24 - P. pseudomystica Zinovjev, 1987. 
Phaonia oxystoma Emden, 1965: 233 (Phaonia)

MATERIAL. ZIN: Paratype, 10': (MYANMAR, Kachin st.), N. E. Burma Kambaiti $\left(25.53^{\circ} \mathrm{N} 98.14^{\circ} \mathrm{E}\right), 2000 \mathrm{~m}$, R. Malaise, 4.06.1934, Phaonia oxystoma sp.n. paratype Van Emden det., 1953; Pres by, Com.Inst.Ent., B.M. 1957-401.

Other type material. NRS: Holotype $\sigma^{7}$, the same locality as the above mentioned paratype, 6.04.1934.

NRS, BMNH and ZMUH: paratypes, $22 \sigma^{\top} \sigma^{7}, 10 \% \circ$, the same locality, 30.04-17.06.1934 [Emden, 1965].

Zoological Survey of India, Calcutta, paratypes: $20^{7} \sigma^{7}, 290$, (INDIA, $W$ Bengal st.), N India, Darjeeeling $\left(27.04^{\circ} \mathrm{N} 88.26^{\circ} \mathrm{E}\right)$ $9000 \mathrm{ft} .=2700 \mathrm{~m}$ asl, E. Brunetti, 27-29.05.1910 [Emden, 1965].

Phaonia oxystomodes Emden, 1965: 237 (Phaonia) Fig. 44.

MATERIAL. ZIN: Paratype, $10^{7}$ : (MYANMAR, Kachin st.), N. E. Burma Kambaiti $\left(25.53^{\circ} \mathrm{N} 98.14^{\circ} \mathrm{E}\right), 2000 \mathrm{~m}$, R. Malaise, 18.04.1934, Phaonia oxystomodes sp.n. paratype Van Emden det., 1953; Pres by, Com.Inst.Ent., B.M. 1957-401.

Other type material. NRS: Holotype $\sigma^{7}$, the same locality as the above mentioned paratype, 24.04.1934.

NRS, BMNH and ZMUH: paratypes, $24 \sigma^{7} \sigma^{\top}, 16$ 우, the same locality, 8.04-17.06.1934 [Emden, 1965].

Phaonia paradecussata Hennig, 1963: 853 (Phaonia) Fig. 25.

MATERIAL. ZIN: Holotype, О’: пер. Анзоб. Гиссар хр. Тадж. Грунин 7.VI.956 (= TAJIKISTAN, Sughd reg., Gissar Range, Anzob pass, $39.08^{\circ} \mathrm{N} 68.87^{\circ} \mathrm{E}, \mathrm{H}^{\prime} 3300 \mathrm{~m}$ asl, 7.06.1956, K. Grunin), Phaonia paradecussata $\sigma^{7}$ Hennig n.sp. Holotypus.

Paratypes $2 \sigma^{7} \sigma^{2}$, TAJIKISTAN: the same label as the holotype, 3.07.1956, $10^{7} ; 11.07 .1956,10^{7}$.

Other type material. According to Hennig's supplementary label on the holotype, $1 \Im^{7}$ paratype and 19 were taken to SDEI. However, the $\sigma^{7}$ paratype was not found neither in SDEI [Rohlfien, Ewald, 1974] nor in ZMHU [Pont, Werner, 2006].

REMARKS. Totally 4 more male paratypes with the same geographical part of the label as the holotype collected on 3, 8, 9 and 11.07.1956 were mentioned in Hennig's [1963] original description. The paratypes from 8 and 9.07.1956 were not found. We found one more $\sigma^{7}$ marked as a paratype (probably by Zinovjev), but it is not a paratype because it was collected on 5.07.1956. There are also several $\sigma^{7} \sigma^{7}$ and $\$ \circ$ specimens without identification label collected by K. Grunin in 1956 from Anzob, they are topotypes of Ph. subdecussata or Ph. paradecussata.

\section{Phaonia pseuderrans Hennig, 1963: 860 (Phaonia)} Fig. 26.

MATERIAL. ZIN: Holotype, $\bigcirc^{7}$ : с. Мангит Кара Каракалпакия Узбекист. 5. IV 1957г Сычевская (= UZBEKISTAN, Karakalpakstan reg., Mangit village, $42.1^{\circ} \mathrm{N} 60.1^{\circ} \mathrm{E}$, V. Sychevskaya, 5.04.1957), Phaonia pseuderrans n.sp. Hennig Holotypus.

Phaonia pseudomystica Zinovjev, 1987: 436 (Phaonia) Fig. 24.

Phaonia mystica Meigen, 1826 sensu Shinonaga \& Kano [1971], misidentification.

MATERIAL. ZIN: Holotype, О': Тигровый, Партизанск. p-н., ю. Приморье Зиновьев 21.VII.1979 (= RUSSIA, Primorsky reg., Partizansky dist., Tigrovyi, $43.844^{\circ} \mathrm{N} 132.772^{\circ} \mathrm{E}$, A. Zinovjev, 21.07.1979), Holotypus Phaonia pseudomystica $O^{7}$ Zinovjev sp.n. 1985.

Paratypes, $3 \sigma^{7} \sigma^{7}, 1900$, RUSSIA, Primorsky reg.: the same label as the holotype, 1 ; ; с. Молчановка, Партизанск. р-н лес, В. Куслицкий (= Partizansky dist., Molchanovka vill., $43.48^{\circ} \mathrm{N}$ $133.36^{\circ} \mathrm{E}$, V. Kuslitsky), 28.06.1972, $10^{7}$;

Анисимовка, Шкот. р-н., Приморье, А.Зиновьев (= Shkotovsky dist., Anisimovka, $43.17^{\circ} \mathrm{N} 132.79^{\circ} \mathrm{E}, \mathrm{A}$. Zinovjev) 22.07.1979, 1; 28.07.1979, 3ㅇ; 11-13.09.1979, 4우 ; Владивосток, Седанка, Зиновьев (= Vladivostok, Sedanka, $43.2^{\circ} \mathrm{N} 132.0^{\circ} \mathrm{E}$,
A. Zinovjev), 15.05.1979, 10; 19.07.1979, 1; Океанская, Владивосток, А.Зиновьев (= Vladivostok, Okeanskaya, $43.26^{\circ} \mathrm{N}$ $132.04^{\circ} \mathrm{E}$, A. Zinovjev), 29.08.1978, 19; Б. Золотой Рогъ., Владив. Прим., обл. Рыдзевскій и Вр. Кузнецовъ (= Vladivostok, Zolotoy Rog Bay, $43.10^{\circ} \mathrm{N} 131.88^{\circ} \mathrm{E}$, Rydzevsky and Kuznetsov), 8.07.1911, 19; зап. Кедровая падь, южн. Приморье, Нарчук (= Kedrovaya Pad Nat. Res., $43.1^{\circ} \mathrm{N} 131.5^{\circ} \mathrm{E}$, E. Narchuk), 20.06.1962, 1 ; 17.08.1962, 1; кл. Нарва, Ю3, п. Барабаш, Хасанск. р-н. Приморье, Каспарян (= Khasansky distr., Barabash vill., $43.2^{\circ} \mathrm{N}$ $131.5^{\circ} \mathrm{E}, \mathrm{D}$. Kasparyan), 4.08.1978, 3우; Горно-таежн. ст., 20 км ЮВ Уссурийска, Нарчук (= $20 \mathrm{~km}$ SE of Ussuriysk, GornoTaiozhnoe, $43.70^{\circ} \mathrm{N} 132.15^{\circ} \mathrm{E}$, E. Narchuk), 2.08.1963, ${ }^{\circ}$; верх. Чапигоу прит., Шуфана ю. Приморье, Нарчук (= upper reaches of Chapigou=Gladkaya River, tributary of Shufan=Borisovka R., $44.0^{\circ} \mathrm{N} 131.6^{\circ} \mathrm{E}$, E. Narchuk), 5.07.1962, 1 영 Amur reg., окр. г. Зеи, Амур. обл., Зиновьев (= Zeya env., $53.7^{\circ} \mathrm{N} 127.2^{\circ} \mathrm{E}$, А. Zinovjev), 23.06.1957, $10^{7}$.

Remarks. Two specimens, $1 \sigma^{\top}, 1+$, listed as paratypes by Zinovjev [1987] were not found, possibly they are in BMNH.

Phaonia pura Loew, 1873: 48 (Aricia)

Fig. 27

MATERIAL. ZIN: Lectotype, $\sigma^{7}$ : Herculan 5.6.871 820 (= ROMANIA, Baile Herculane, $44.88^{\circ} \mathrm{N} 22.41^{\circ} \mathrm{E}, 5.06 .1871$ ), sec. type. Loew. Ar pura Lw Kowarz det. Lectotype $\sigma^{7}$ Aricia pura Loew Des Zinovjev 1987.

Phaonia rufitarsis Stein, 1907: 321 (Aricia)

REMARKS. According to Pont [2013]: holotype $\sigma^{7}$, seen by Hennig [1963: 864] but not now in ZIN and not seen by A. Zinovjev.

\section{Phaonia sibirica Pont, 1981: 427 (Phaonia)} Fig. 28

MATERIAL. ZIN: Holotype, О’: Низ. Оби 15. VII 1973 Лабытнанги В. Сычевская; цветы шиповника (= RUSSIA, YamaloNenets reg., Labytnangi, $66.66^{\circ} \mathrm{N} 66.38^{\circ} \mathrm{E}$, on dog rose flowers, V. Sychevskaya, 15.07.1973), Holotype $\sigma^{7}$ Phaonia sibirica sp.n. A. C. Pont.

Other type material. BMNH, 19 paratype [Pont, 1981]: RUSSIA, the same label as the holotype, date 4.07.1973.

\section{Phaonia sichotensis Zinovjev, 1980: 910 (Phaonia)} Fig. 37.

MATERIAL. ZIN: Holotype, О’: Анисимовка (Кангауз) Шкотовск. р-н, ю. Приморье Зиновьев 23.VI.1979; широкол. и хвойно-широкол. лес; (= RUSSIA, Primorsky reg., Shkotovsky dist., Anisimovka, $43.17^{\circ} \mathrm{N} 132.79^{\circ} \mathrm{E}$, A. Zinovjev, 22.6.1979), Holotypus Phaonia sichotensis $\sigma^{7}$ Zinovjev, 1980.

Paratypes $10^{7}, 1+$, RUSSIA, the same label as the holotype, date 22.06.1979.

\section{Phaonia splendida Hennig, 1963: 872 (Phaonia)} Fig. 29.

MATERIAL. ZIN: Holotype, $\sigma^{7}$ : пер. Анзоб. Гиссар. хр., Тадж Грунин 22.VI.956 (= TAJIKISTAN, Sughd reg., Gissar Range, Anzob pass, $39.08^{\circ} \mathrm{N} 68.87^{\circ} \mathrm{E}, \mathrm{H}^{\prime} 3300 \mathrm{~m}$ asl, K. Grunin, 22.06.1956), Phaonia splendida n.sp. Hennig Holotypus.

Paratypes $2 \sigma^{7} \sigma^{7}$, TAJIKISTAN: the same label as the holotype, but date 23-24.06.

Other type material. According to Hennig's supplementary label on the holotype 1 specimens was taken to SDEI, it should be O paratype with the the same label as the holotype. However, this $\sigma^{7}$ paratype was not found neither in SDEI [Rohlfien, Ewald, 1974] nor in ZMHU [Pont, Werner, 2006].

\section{Phaonia stackelbergi Hennig, 1963: 873 (Phaonia)} Fig. 30

MATERIAL. ZIN: Holotype, O’: ур. Квак, верх. p. Кондары, Тадж. Штакельберг 7.VI.43 (= TAJIKISTAN, Sughd reg., upper reaches of Kondara R., Kvak tract, $38.81^{\circ} \mathrm{N} 68.81^{\circ} \mathrm{E}, \mathrm{H}$ " $1300 \mathrm{~m}$ asl, 


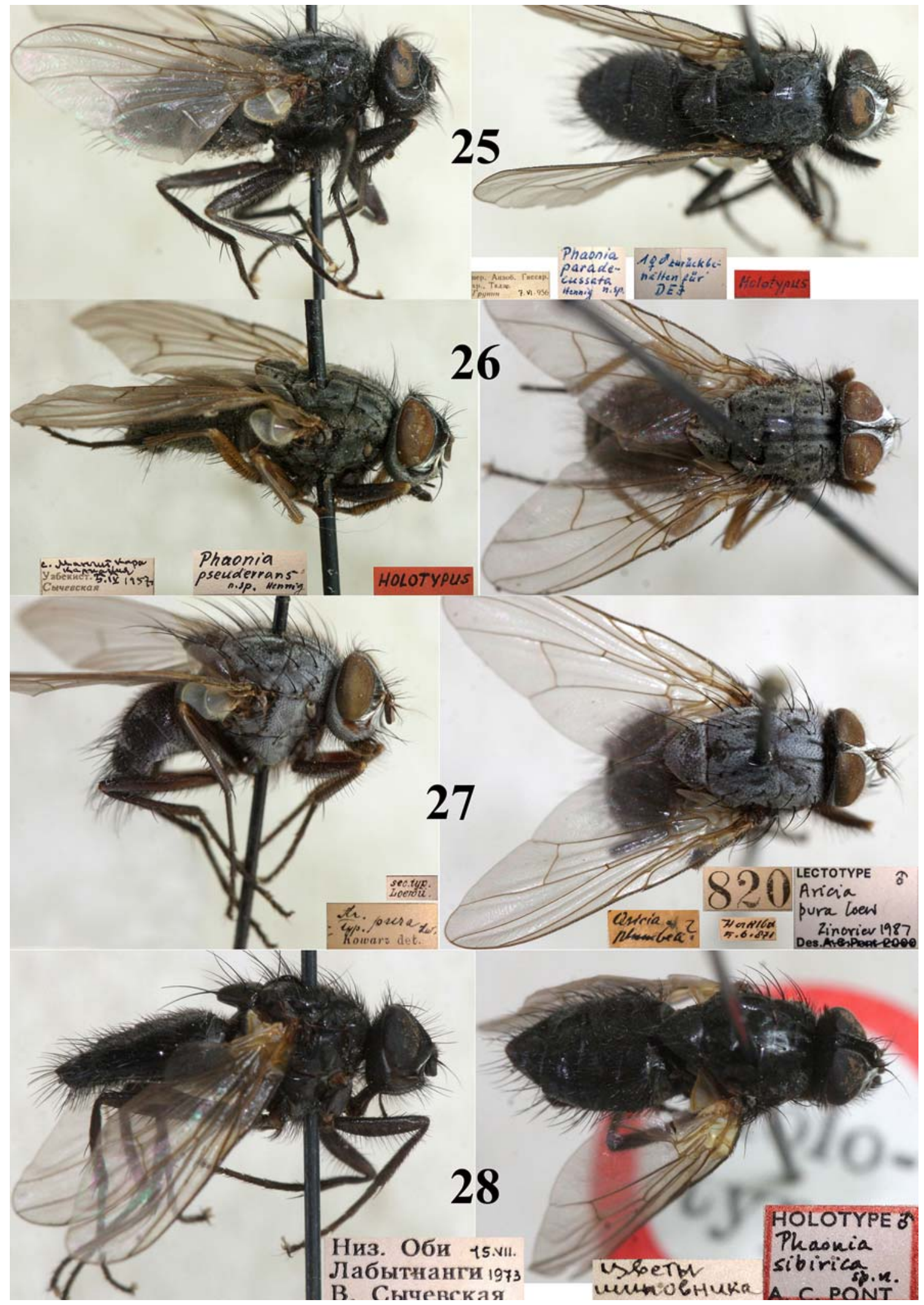

Figs 25-28. Phaonia spp., $O^{7}$ holotypes: 25 - P. paradecussata Hennig, 1963; 26 - P. pseuderrans Hennig, 1963; 27 - P. pura Loew, 1873; $28-$ P. sibirica Pont, 1981.

Рис. 25-28. Phaonia spp.:, О7 голотип: 25 - P. paradecussata Hennig, 1963; 26 - P. pseuderrans Hennig, 1963; 27 - P. pura Loew, 1873; $28-$ P. sibirica Pont, 1981. 
A. Stackelberg, 7.06.1943), Phaonia stackelbergi Hennig n.sp. Holotypus.

Paratypes $20^{7} 0^{7}$, TAJIKISTAN, Sughd reg: Зидды, Ю скл, Гиссарск. хр., Тадж. Штакельберг (= S slope of Gissar Range, Ziddy R., $38.81^{\circ} \mathrm{N} 68.81^{\circ} \mathrm{E}, 2000 \mathrm{~m}$ asl, A. Stackelberg, 14 and 16.06.1944)

Other type material. According to Hennig's supplementary label on the holotype $1 O^{7}$ specimens was taken to SDEI, but all type material mentioned in the original description was found in ZIN.

Phaonia subcandicans Zinovjev, 1983: 188 (Phaonia)

Fig. 31.

MATERIAL. ZIN: Holotype, $\sigma^{7}: 30$ км вост. Нарын ТяньШань 2600-800 Нарчук 7. VIII.969 (= KYRGYZSTAN, Tian Shan, $30 \mathrm{~km}$ E of Naryn, $41.44^{\circ} \mathrm{N} 76.36^{\circ} \mathrm{E}, 2600 \mathrm{~m}$ asl, E. Narchuk, 7.08.1969), Holotypus Phaonia subcandicans $0^{7}$ Zinovjev, 1982.

Paratypes, $30^{7} 0^{7}, 3$ 우, KYRGYZSTAN: Терскей Алатоо, Чон Кызыл-су H"2200, Второв (= Terskey Alatau, Chon-Kyzyl-Su River, $42.26^{\circ} \mathrm{N} 78.12^{\circ} \mathrm{E}, 2200 \mathrm{~m}$ asl, P. Vtorov), $20.05 .1965,10^{7}$; 30 км Ю Фрунзе, Кашкасу 1700 м, Каспарян (= $30 \mathrm{~km} \mathrm{~S}$ of Bishkek=Frunze, Kashka-Su, $42.68^{\circ} \mathrm{N} 74.55^{\circ} \mathrm{E}, 1700 \mathrm{~m}$ asl, D. Kasparyan), 13.06.1979, $20^{7} \sigma^{7}, 3$ 우우.

\section{Phaonia subdecussata Hennig, 1963: 875 (Phaonia)} Fig. 32.

MATERIAL. ZIN: Holotype, $0^{7}$ : пер. Анзоб. Гиссар. хр. Тадж. Грунин 24.VI.956; Норы сурков (= TAJIKISTAN, Sughd reg., Gissar Range, Anzob pass, $39.08^{\circ} \mathrm{N} 68.87^{\circ} \mathrm{E}, \mathrm{H}^{\prime \prime} 3300 \mathrm{~m}$ asl, marmot burrows, K. Grunin, 24.06.1956), Holotypus Phaonia subdecussata $\sigma^{7}$ Hennig n. sp.

Paratypes, $4 \bigcirc^{7} \sigma^{7}$, TAJIKISTAN: the same label as the holotype, 23.06.1956, $10^{7} ; 24.06 .1956,20^{7} O^{7} ; 26.06 .1956,10^{7}$.

Other type material. According to Hennig's supplementary label on the holotype $1 \sigma^{7}$ specimens was taken to SDEI, but all the type material mentioned in the original description was found in ZIN.

\section{Phaonia suspiciosa Stein, 1907: 324 (Spilogaster)} Fig. 33.

Phaonia fusca suspiciosa Zinovjiev, 1990: 476

Phaonia fusca Meade, 1897 = Phaonia suspiciosa Stein, 1907 [Vikhrev, Sorokina, 2017].

MATERIAL. ZIN: Lectotype, + : p Орогын Сыртын ю Наньшаня Гоби Роб Козлов 3-20.VII.95 (= CHINA, border of Xinjiang and Qinghai prov., S of Nanshan=Qilian Mts., H" $39^{\circ} \mathrm{N} 94^{\circ} \mathrm{E}, \mathrm{V}$. Roborovsky, P. Kozlov, 3-20.07.1895), Spilogaster suspiciosa Stein, Lectotypus Spilogaster suspiciosa Stein 1907 design. Zinovjev, 1979.

Paralectotypes, $23 \sigma^{7} \sigma^{\top}, 7+9$. CHINA: the same label as the lectotype, $60^{\top} \sigma^{7}, 3$ ㅇ; p. Дза-чю, 11000' Камъ, бас. Голубой Козлов (= Sichuan prov., $\mathrm{H}^{\prime \prime} 32.0^{\circ} \mathrm{N} 99.5^{\circ} \mathrm{E}, 11000 \mathrm{ft} .=3350 \mathrm{~m}$ asl, P. Kozlov), mid April 1901, $10^{7}$; р Бомын (Ичегын) св Цайдамъ, Гоби Роб Козлов кVI95 (= Oinghai prov., NE Tsaidam, Gob desert, Ichegyn River, $38.0^{\circ} \mathrm{N} 94.8^{\circ} \mathrm{E}, 3100 \mathrm{~m}$ asl, V. Roborovsky, P. Kozlov), end of June 1895, $8 \sigma^{7} \sigma^{\top}, 3$ 우; Хабирга-оз. Бага цадамин, в. Цайд Роб Козлов (= Qinghai prov., E Tsaidam, Khabirga, Baga-tsaidamin Lake, $37.6^{\circ} \mathrm{N} 95.4^{\circ} \mathrm{E}, 3200 \mathrm{~m}$ asl, V. Roborovsky, P. Kozlov), 3-11.06.1895, $40^{\top} \sigma^{\top}, 1$ 우 х. Барун-Цзасака мет.ст., в Цайдам Козлов. кон. VII01 (= Qinghai prov., E Tsaidam, $36.2^{\circ} \mathrm{N}$ $97.4^{\circ} \mathrm{E}, 2800 \mathrm{~m}$ asl, P. Kozlov), end of July.1901, $3 \sigma^{7} \sigma^{7}$; Курлык, Баингол вост. Цайдамъ Роб Козлов (= Qinghai prov., Е Tsaidam, Kurlyk=Keluke Lake, Baingol River, $37.3^{\circ} \mathrm{N} 96.9^{\circ} \mathrm{E}, 2850 \mathrm{~m}$ asl, V. Roborovsky, P. Kozlov), 28.05.1895, $1 \sigma^{7}$.

Other type material. ZMHU, paralectotypes, $4 \sigma^{7} \sigma^{\top}, 19$. BMNH, paralectotype, $10^{7}$ [Pont, Werner, 2006].

REMARKS. This species is represented by large type series collected by Russian geographical expeditions in Central Asia in 1893-1895 by V. I. Roborovsky and P. K. Kozlov [Roborovsky, 1949] and in 1899-1901 by P. K. Kozlov [Kozlov, 1947]. The localities of the type specimens were shortly mentioned in Stein's [1907] original description, but the number of specimens was not provided. So did Hennig [1963].

Zinovjev [1990] regarded this species as a subspecies of P. fusca Meade, 1897, Phaonia fusca suspiciosa Stein, 1907. According to Zinovjev's opinion, the structure of the female ovipositors is more useful character than the male terminalia. That was probably the reason why he designated as the lectotype the female specimen [Zinovjev, 1990] (not a male as indicated by Pont and Werner [2006]).

\section{Phaonia sytschevskajae Hennig, 1963: 877} (Phaonia)

MATERIAL. ZIN: Holotype, $\sigma^{7}$ : Джиргитальск. p-н Таджикистан, ур. Каратанг Сычевская 11.VI.947; цв. Ferula, (= TAJIKISTAN, Republican Subordination reg., Jirgatol dist., Karaton tract, $39.19^{\circ} \mathrm{N} 71.27^{\circ} \mathrm{E}, 1800 \mathrm{~m}$ asl, flowers of Ferula, V. Sychevskaya, 11.06.1947), Holotypus; Phaonia sytchevskayae n.sp. Hennig.

Paratype, 19 , KAZAKHSTAN, Almaty reg., Горельник р. М. Алмаатинка, Заилиск. Алатау, кал. В. Сычевская (= Trans-Ili Alatau range, M Almaatinka R., Gorelnik gorge, $43.135^{\circ} \mathrm{N} 77.062^{\circ} \mathrm{E}$, $2100 \mathrm{~m}$ asl, faeces, V. Sychevskaya, 17.08.1947).

\section{Phaonia taigensis Zinovjev, 1987: 440 (Phaonia)} Fig. 34

MATERIAL. ZIN: Holotype, $\sigma^{7}$ : Вырица, под. корой ели Ленингр. обл. 1 2.V.79 im 19.V.1979, Зиновьев (= RUSSIA, StPetersburg reg., Vyritsa, $59.4^{\circ} \mathrm{N} 30.3^{\circ} \mathrm{E}$, , A. Zinovjev, from pupa found under fir bark 2.05.1979, ex pupa 19.05.1979), Holotypus Phaonia taigensis $\sigma^{7}$ Zinovjev sp.n. 1985.

Paratypes, $140^{\top} 0^{7}, 23$ ㅇ․ RUSSIA, St-Petersburg reg.: the same label as the holotype, but reared $15-19.05 .1980$ or 1822.05.1980, 6өо; Семрино, 45 км Ю Ленинграда, под корой ели, 22.IV, im. 15-18.V.1980, Зиновьев $(=45 \mathrm{~km} \mathrm{~S}$ of St-Petersburg, Semrino, $59.54^{\circ} \mathrm{N} 30.38^{\circ} \mathrm{E}$, A. Zinovjev), from pupa found under fir bark 22.04.1980, imago emerged 15-18.05.1980, $2 \sigma^{\top} \sigma^{\top}, 3$, 90 ; Amur reg., верх. Р. Эракингры Тукурингра, Амур. долинный ельник Зиновьев (= Tukuringra Range, upper reaches of Erakingra River, $54.1^{\circ} \mathrm{N} 126.9^{\circ} \mathrm{E}$, fir forest, A. Zinovjev), 21.06-2.07.1957, $2 \sigma^{\top} \sigma^{7}$, 290; Arkhangelsk reg., г. Архангельск, из ходов типогр., лич. 11.VI и 20.VI, имаго 25.VI.1972 (= Arkhangelsk, $64.5^{\circ} \mathrm{N} 40.6^{\circ} \mathrm{E}$, from tunnels of Ips typographus in fir, B. Ogibin), pupa found 20.06.1972, imago emerged 25.06.1972, $1 \sigma^{7}$; larva found 25.06.1972, imago emerged 11.07.1972, 1; Perm reg., Кунгур, Пермск. (Молотовская) обл., учлесхоз, лич под корой срубленной ели, К.Борисова, Зиновьев (= Kungur, Uchleskhoz=Preduralie $\mathrm{NP}$, presently abolished, $57.36^{\circ} \mathrm{N} 57.14^{\circ} \mathrm{E}$ ), from larvae under the bark of felled fir, A. Zinovjev, imago emerged 7-19.06.1955, $2 \sigma^{7} \sigma^{7}$, 1; from larva from litter under fir, K. Borisova, imago emerged 7.06.1956, 19; Vologda reg., Вологодская обл. Усть-Кубенск. p. Никольское 7.6.83 п/к ели, в 6.8 НКрив (= Ust-Kubensk distr, Nikolskoe, $59.85^{\circ} \mathrm{N} 39.36^{\circ} \mathrm{E}$, N. Krivosheina), from pupa found

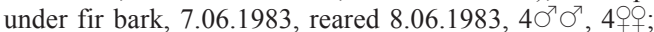

FINLAND, Финляндия, окр.Хельсинки, п/к осины, Б.Мамаев (= Helsinki env, reared from pupa found under aspen bark, В. Mamaev), $10^{7}, 1$ \%

KYRGYZSTAN, Issyk-Kul reg., Киргизия Иссык-кульская обл. Теплоключенка, под корой тянь-шанской ели (= Teploklyuchenka $=\mathrm{Ak}-\mathrm{Suu}, 42.5^{\circ} \mathrm{N} 78.5^{\circ} \mathrm{E}$, from pupa found under the bark of Picea schrenkiana subsp. Tianschanica), $20^{\top} \sigma^{\top}, 4+9$.

Other type material. ZMUM, paratypes $10^{7}, 1$, RUSSIA, the same label as the holotype.

\section{Phaonia tenuirostris Stein, 1907: 320 (Aricia)}

$$
\text { Fig. } 35 .
$$

MATERIAL. ZIN: Lectotype, $\sigma^{7}$ : оз. исток Хуан хэ, с.-в. Тибетъ Козловъ 14.VI.900 (= CHINA, Qinghai prov., NE Tibet, source of Huang He R., Ngoring Lake or Gyaring Lake, $34.8^{\circ} \mathrm{N}$ $97.5^{\circ} \mathrm{E}, 4270 \mathrm{~m}$ asl, P.K. Kozlov, 14.06.1900); Lectotype $\sigma^{\top}$ Aricia tenurostris Stein Des.A.C.Pont 2000.

Paralectotypes $10^{7}, 6$ 우: CHINA, the same label as the lectotype. 


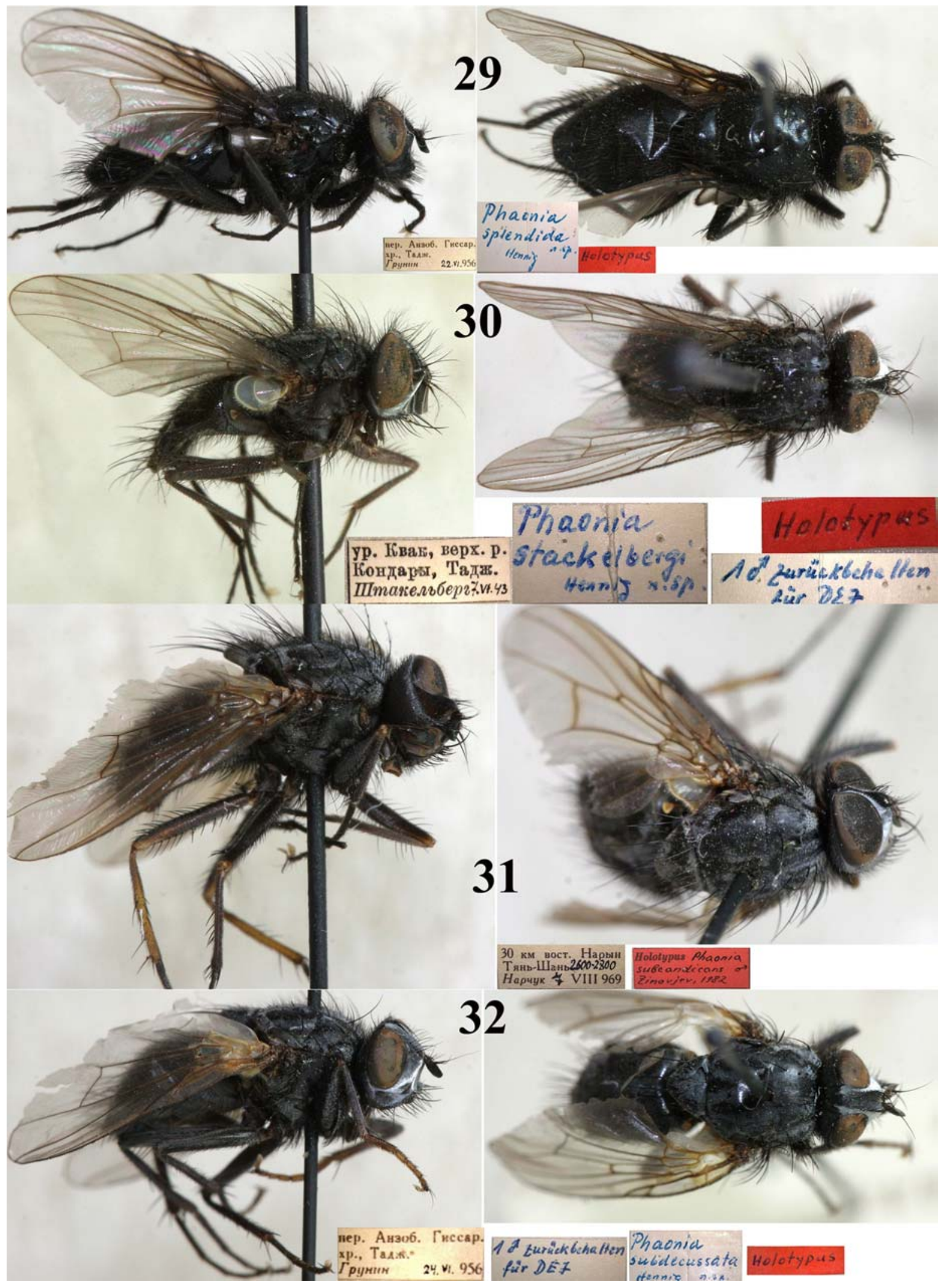

Figs 29-32. Phaonia spp., O’ holotype: 29 - P. splendida Hennig, 1963; $30-$ P. stackelbergi Hennig, 1963; 31 - P. subcandicans Zinovjev, 1983; $32-P$. subdecussata Hennig, 1963.

Рис. 29-32. Phaonia spp., О7 голотипы: 29 - P. splendida Hennig, 1963; 30 - P. stackelbergi Hennig, 1963; 31 — P. subcandicans Zinovjev, 1983; 32 - P. subdecussata Hennig, 1963. 


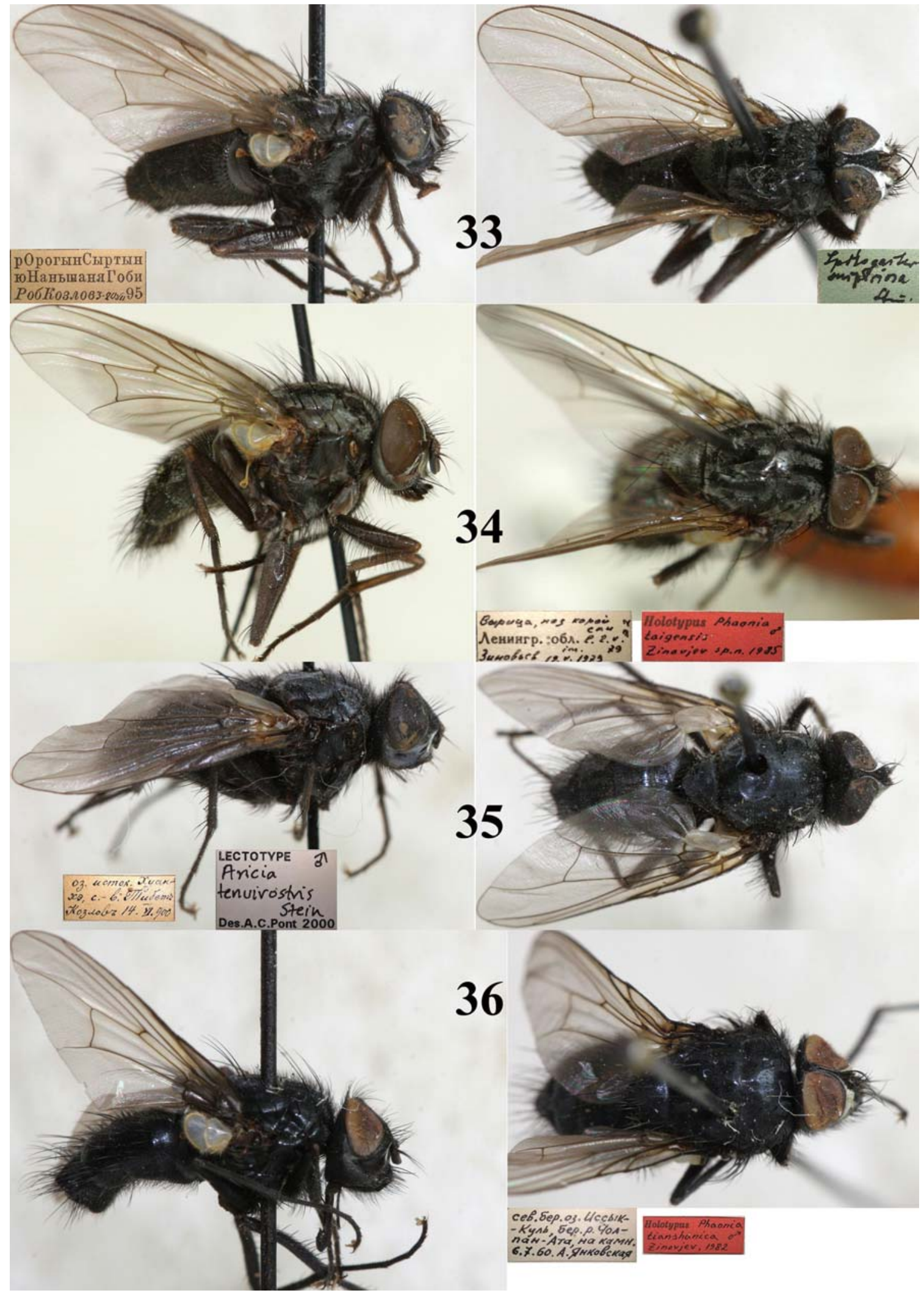

Figs 33-36. Phaonia spp.: $33-P$. suspiciosa Stein, 1907, $\sigma^{7}$ paratype; $34-P$ taigensis Zinovjev, 1987, $O^{7}$ holotype; $35-P$ tenuirostris Stein, 1907, O holotype; $36-P$. tianshanica Zinovjev, 1983, $\sigma^{7}$ holotype.

Pис. 33-36. Phaonia spp.: 33 - P. suspiciosa Stein, 1907, O' паратип; $34-P$. taigensis Zinovjev, 1987, О' голотип; $35-P$. tenuirostris Stein, 1907, О7 голотип; 36 - P. tianshanica Zinovjev, 1983, О7 голотип. 
Other type material. ZMHU. Paralectotypes, $10^{7}, 3 \circ+$, CHINA, the same label as the lectotype [Hennig, 1963; Pont, Werner, 2006].

\section{Phaonia tianshanica Zinovjev, 1983: 190 (Phaonia) Fig. 36.}

MATERIAL. ZIN: Holotype, $\sigma^{7}$ : сев. бер. оз. Иссык-Куль, бер. p. Чолпан-Ата, на камн. 6.7.60. А. Янковская (= KYRGYZSTAN, Issyk Kul reg., N shore of Issyk Kul Lake, bank of Cholpon-Ata R., $42.66^{\circ} \mathrm{N} 77.08^{\circ} \mathrm{E}$, on stones, A. Yankovskaya, 6.07.1960). Holotypus Phaonia tianshanica OT Zinovjev, 1982.

Paratypes, $10 \sigma^{7} \sigma^{7}, 16$ + 9 . KAZAKHSTAN, Семиреченск. обл., дол. p. Коры, Шнитников (= Semirechye Oblast of Russian Empair, presently Almaty reg., Kora River Valley, $45.0^{\circ} \mathrm{N} 79.0^{\circ} \mathrm{E}, \mathrm{V}$. Shnitnikov), 23.07.1908, 1웅

KYRGYZSTAN: the same label as the Holotypes, $1 \sigma^{7}$; Терскей Алатоо, Чон. Кызыл-су, Второв (=Terskey Alatau Range, Chong Kyzyl-Suu valley, $\mathrm{H}^{\prime} 42.22^{\circ} \mathrm{N} 78.17^{\circ} \mathrm{E}, \mathrm{P}$. Vtorov): H” $2200 \mathrm{~m}$ asl, 20.05.1965, $1 \mathrm{O}^{7}$; H" $2600 \mathrm{~m}$ asl, on faeces, 12.07.1965, 19; xp. Атбаши 40 км ЮВ, г. Нарын 2700-3500м, В. Зайцев (= 40 km SE of Naryn, At-Bashi Range, H"41.25 ${ }^{\circ} \mathrm{N} 76.40^{\circ} \mathrm{E}, 2700-3500 \mathrm{~m}$ asl, V. Zaitsev), 14.07.1966, 2о; ущ. Аламедин, 30 км, S Фрунзе, степь, В. Зайцев (=30 km S of Bishkek, Alamedin Valley, $42.60^{\circ} \mathrm{N}$ $74.66^{\circ} \mathrm{E}$, steppe, V. Zaitsev), 2.07.1966, $2 \sigma^{7} \sigma^{7}$; Сусамыр. дол. Кирг., 30 км W Сусамыра, В. Зайцев (= Susamyr River Valley, 30 $\mathrm{km} \mathrm{W}$ of Susamyr Town, $42.21^{\circ} \mathrm{N} 73.67^{\circ} \mathrm{E}$, V. Zaitsev), 29.06.1966, $10^{7}$; у ледн. Совнаркома, ист. Нарына. Семир., Кашкаров (= Semirechye or Zhetysu, source of Naryn River, $41.77^{\circ} \mathrm{N} 77.97^{\circ} \mathrm{E}$, D. Kashkarov), 23.07.1934, $2 \sigma^{7} \sigma^{7}, 1$ 우 ней, 2700мт (=Erkeshtam = Irkeshtam, border (with China), $39.68^{\circ} \mathrm{N}$ $73.90^{\circ} \mathrm{E}, 2700 \mathrm{~m}$ asl, N. Olsufiev), 16.07.1935, 10, 1; ущ. p. Туюк, Киргизия, Шнитников, (= Tuyuk River Valley, V. Shnitnikov), 4.08.1930, 2कo; Киргизская A.C.C.P, Каракольск. Кантон., ур. Арал-дуб, Сер. Тарб (= Karakol env., H" $42.4^{\circ} \mathrm{N}$ $78.4^{\circ} \mathrm{E}, \mathrm{S}$. Tarbinsky), 15.06.1925, 1 \%; Терскей Алатау, ю. Каракола, Кашкаров (= Terskey Alatau, S of Karakol, H"42.3으 $78.5^{\circ} \mathrm{E}$, D. Kashkarov), 17-22.07.1934, 1; Джеты-огуз, вост., бер. Иссык-куля, М. Берг (= E shore of Issyk-Kul Lake, Jeti Oguz, $42.32^{\circ} \mathrm{N}$ $\left.78.25^{\circ} \mathrm{E}\right), 13.06 .1930,1$;

MONGOLIA, MOНГОЛИЯ, Гоби-Алт., аймак, 15 км ВСВ Цогта, Зайцев (= Govi-Altai prov., $15 \mathrm{~km}$ EEN of Tsogt, $45.4^{\circ} \mathrm{N}$ $96.8^{\circ} \mathrm{E}, \mathrm{V}$. Zaitsev), 15.07.1970, 1웅

TAJIKISTAN: В. Памир, окр. Чечекты, Сычевская (= GornoBadakhshan reg., E Pamir, Chechekty env., $38.34^{\circ} \mathrm{N} 74.01^{\circ} \mathrm{E}, \mathrm{V}$ Sychevskaya (Porutek and Mukor River are localities near Chechekty, we can not give a more accurate definition)): Порутек, помет сурка (marmot's dung) $3800 \mathrm{~m}$ asl, 9.08.1965, 107' p. Мукор, на камнях (on stones), $4300 \mathrm{~m}$ asl, 1.08.1962, 10', 1; 11.08.1964, 1ㅇ $\mathrm{p}$. Порутек , $4100 \mathrm{~m}$ asl, 18.08.1965, 10; В. Памир, с Мордж, Таджик. Н"3400, Сычевская (= Gorno-Badakhshan reg., Mardzh, $37.70^{\circ} \mathrm{N} 72.45^{\circ} \mathrm{E}, 3400 \mathrm{~m}$ asl, V. Sychevskaya), 4.08.1964), 1 ㅇ․

Other type material. ZMUM: paratype $1+$, KYRGYZSTAN, Тянь-Шань, р. Куйлю, П. Второв (= Tian Shan, Kuylu R., H' $42.4^{\circ} \mathrm{N} 78.5^{\circ} \mathrm{E}, \mathrm{P}$. Vtorov), 30.06.1962.

BMNH: paratype $10^{7}$, NEPAL, Baitadi, Tinkar Khola $\left(29.52^{\circ} \mathrm{N}\right.$ 80.49² E), J. B. Tyson, 3.07.1953, [Zinovjev, 1983].

REMARKS. Three more females from Kyrgyzstan, Terskey Alatau, S of Karakol, D. Kashkarov were marked as the paratypes, but these 3 + 0 were not mentioned in the original description.

Phaonia ussuriensis Zinovjev, 1980b: 908 (Phaonia) Fig. 38.

MATERIAL. ZIN: Holotype, $\sigma^{7}$ : Владивосток Седанка Зиновьев 17.VII.1979 Holotypus $\sigma^{7}$ Phaonia ussuriensis Zinovjev, 1980 (= RUSSIA, Primorsky reg., Vladivostok, Sedanka, $43.2^{\circ} \mathrm{N} 132.0^{\circ} \mathrm{E}$, A. Zinovjev 17.07.1979).

Paratypes $24 \sigma^{7} \sigma^{7}, 21$ 우. RUSSIA, Primorsky reg.: the same label as the holotype, data: 25.08.1978, 200; 17, 19 and 25.07.1979, $7 \sigma^{7} \sigma^{7}, 9$ qо; Тигровый, Партизанск. р-н., ю. Приморье Зиновьев (= Tigrovyi, $43.844^{\circ} \mathrm{N} 132.772^{\circ} \mathrm{E}$, A. Zinovjev), 21-22.07.1979, $4 \sigma^{\top} \sigma^{7}, 3$ о; зап. Кедровая падь южн. Приморье Нарчук кордон Геккелев ключ, (= Kedrovaya Pad Nat.Reserve, $43.1^{\circ} \mathrm{N} 131.5^{\circ} \mathrm{E}$,
E. Nartchuk), 6.08.1962, 1; Владивосток Академгородок В.Куслицкий разнотравье и кустарн. под пологом широколиствен. леса (= Vladivostok, Akademgorodok, 43.20 ${ }^{\circ} \mathrm{N} 131.93^{\circ} \mathrm{E}$, herbage and bushes under the broad-leaved forest canopy, V. Kuslitsky), 1020.07.1972, $4 \sigma^{\top} \sigma^{\top}, 2$, цы южн. Приморье В.Куслицкий долина: ива, ольха, лещина (= Khasansky distr., Troitsy Bay, Andreevka village, $42.64^{\circ} \mathrm{N} 131.13^{\circ} \mathrm{E}$, valley: willow, alder, hazel V. Kuslitsky), 12-16.07.1972, $7 \sigma^{7} \sigma^{7}$, 2 우 Анисимовка (Кангауз) Шкотовск, р-н., ю. Приморье Зиновьев (= Shkotovsky dist., Anisimovka env., $43.17^{\circ} \mathrm{N} 132.79^{\circ} \mathrm{E}, \mathrm{A}$. Zinovjev), 22.07.1979, $2 \sigma^{\top} \sigma^{7}, 2$, $9+$.

Other type material. ZMUM: paratypes, $2 \sigma^{7} \sigma^{7}$, RUSSIA, Primorsky reg.: зап. Кедровая падь, Зимина (= Kedrovaya Pad Nat.Reserve, $43.1^{\circ} \mathrm{N} 131.5^{\circ} \mathrm{E}, \mathrm{L}$. Zimina), 7.07.1962, $1 \sigma^{\top}$; Анисимовка (Кангауз) Шкотовск, р-н., ю. Приморье Зиновьев (= Shkotovsky dist., Anisimovka env., $43.17^{\circ} \mathrm{N} 132.79^{\circ} \mathrm{E}, \mathrm{A}$. Zinovjev), 27-28.07.1979, $10^{7}$.

In the original description [Zinovjev, 1980b] 5 more paratypes $\left(4 \Im^{\top} \sigma^{\top}, 1+\right)$ were mentioned, probably they are in some other collection(s).

Phaonia zhelochovtsevi Zinovjev, 1980b: 905 (Dialyta)

Fig. 40.

MATERIAL. ZIN: Holotype, О’: окр. С. Яковлевки ю. Приморье Борисова 15.VI.962, Разреженный дубово-липовый лес с лещиной и леспедецей (= RUSSIA, Primorsky reg., Yakovlevka village env., $44.425^{\circ} \mathrm{N} 133.480^{\circ} \mathrm{E}$, sparse oak-lime forest with hazel Corylus sp. and Lespedeza bicolor, K. Borisova, 15.06.1962), Holotypus Dialyta zhelochovtsevi $\sigma^{7}$ Zinovjev, 1980.

Paratypes 107, 3+o: RUSSIA, Primorsky reg.: Анисимовка (Кангауз) Шкотовск, р-н., ю.Приморье Зиновьев (= Shkotovsky dist., Anisimovka env., $43.17^{\circ} \mathrm{N} 132.79^{\circ} \mathrm{E}$, A. Zinovjev), 22.06.1979, $10^{7}$; Тигровый, Партизанск. р-н., ю. Приморье Зиновьев (= Tigrovyi, $43.844^{\circ} \mathrm{N} 132.772^{\circ} \mathrm{E}$, А. Zinovjev), 22.07.1979, 2оㅇ; Владивосток, Седанка Зиновьев (= Vladivostok, Sedanka, $43.2^{\circ} \mathrm{N}$ 132.0 ${ }^{\circ}$ E, A. Zinovjev), 25.07.1979, 1 ㅇ.

Other type material. ZMUM, paratype: $1^{\circ}$, RUSSIA, Primorsky reg., Спасск-Дальний, Желоховцев (= Spassk-Dalny, $44.6^{\circ} \mathrm{N}$ $132.8^{\circ} \mathrm{E}$, A. Zhelochovtsev), 29.06.1961.

\section{Phaonia zinovjevi Malianov, 1993: 419 (Phaonia)} Fig. 39.

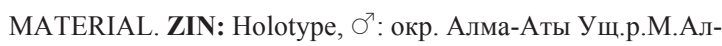
ма-Атинка 1900, в траве Малянов 2.VII.988 (= KAZAKHSTAN, Almaty reg., Zailiysky Alatau Range, canyon of M. Almatinka R., $43.143^{\circ} \mathrm{N} 77.067^{\circ} \mathrm{E}, 1900 \mathrm{~m}$ asl, in grass, M. Malyanov, 2.07.1988), Голотип, all legs on the left side are absent.

Paratypes $1 \sigma^{7}, 1$ 연 KAZAKHSTAN, Almaty reg.: the same label as the holotype, 1 ; ; окр. Тургень 60 км В Алма-Ата Городков (= Turgen env., $43.35^{\circ} \mathrm{N} 77.60^{\circ} \mathrm{E}, \mathrm{K}$. Gorodkov), $15.07 .1969,10^{7}$.

Other type material. Institut of Zoology Academy of Sciences of Kazakhstan, Almaty, Al-Farabi str., 93: paratype, 10', KAZAKHSTAN, the same label as the holotype [Malyanov, 1993].

\section{Supplement}

\section{Specimens marked as types, but apparently not being types.}

Phaonia dorsolineata Shinonaga et Kano, 1971: 171.

MATERIAL. ZIN: JAPAN: Mt. Hayachine IWATE 17-18 June 1971 S. Shinonaga Phaonia dorsolineata Shinonaga et Kano new species, 1971, 1+; Hananoego Yakushima Is. 26-27 Jul 1974 S. Shinonaga Phaonia dorsolineata Shinonaga et Kano new species, $1971,1 \sigma^{7}$.

REMARKS. According to the original description [Shinonaga, Kano, 1971] these specimens were collected 1 or 4 years later than the type series, so the words "new species" on the labels are an error. 




Figs 37-39. Phaonia spp.: 37 - P. sichotensis Zinovjev, 1980, O paratype; 38 - P. ussuriensis Zinovjev, 1980, O' holotype; $39-$ P. zinovjevi Malianov, 1993, $0^{7}$ holotype.

Рис. 37-39. Phaonia spp.: 37 - P. sichotensis Zinovjev, 1980, О' голотип; 38 - P. ussuriensis Zinovjev, 1980, О голотип; 39 P. zinovjevi Malianov, 1993, О голотип. 


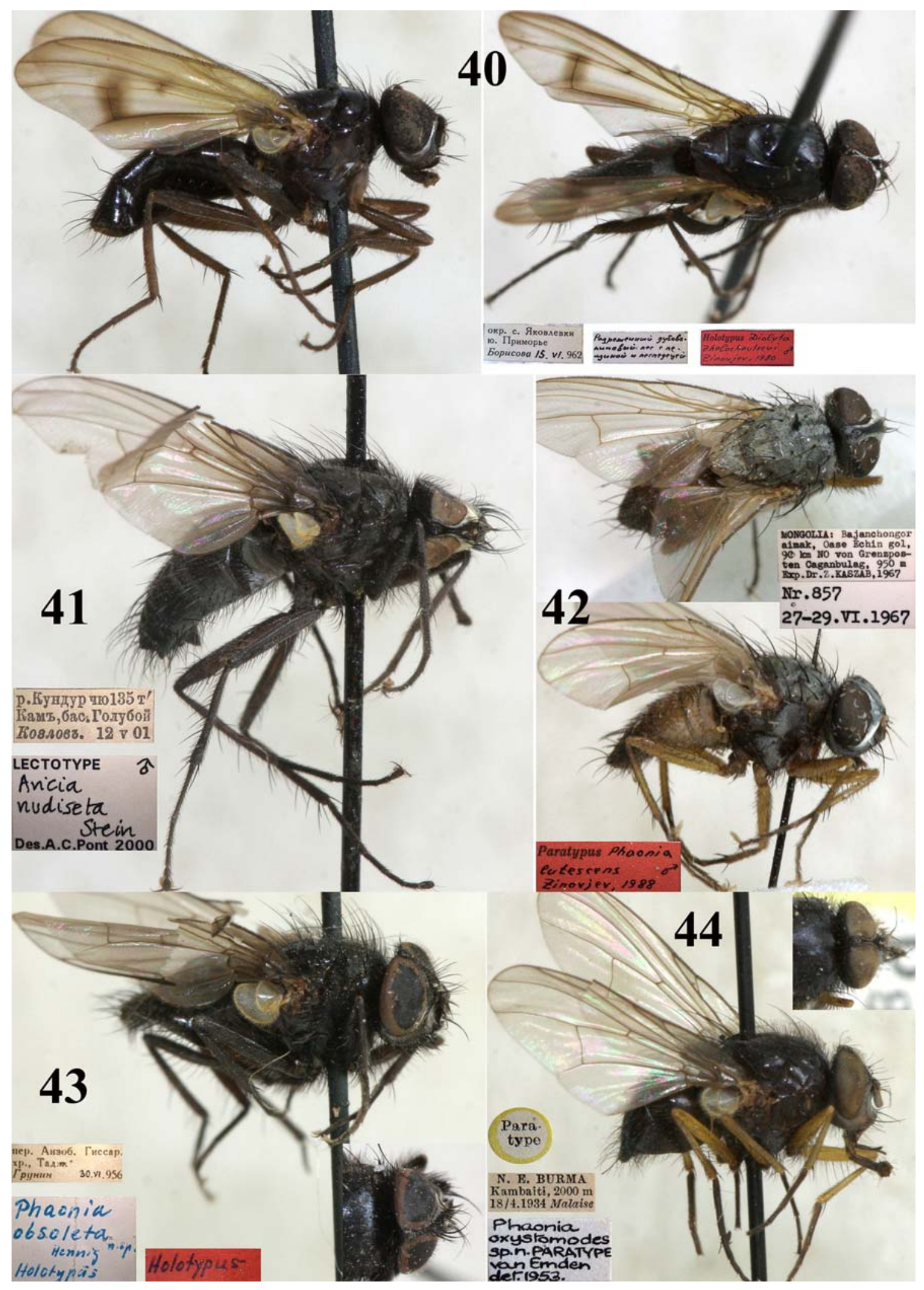

Figs 40-44. Phaonia spp.: $40-P$. zhelochovtsevi Zinovjev, 1980, $\sigma^{7}$ holotype; $41-P$. nudiseta Stein, 1907, $0^{7}$ holotype; $42-P$. lutescens Zinovjev, 1990, Oフ paratype; $43-P$. obsoleta Hennig, 1963, $O^{\top}$ holotype; $44-P$. oxystomodes Emden, 1965, $O^{7}$ paratype.

Рис. 40-44. Phaonia spp.: $40-P$. zhelochovtsevi Zinovjev, 1980, О7 голотип; $41-P$. nudiseta Stein, 1907, О7 голотип; $42-P$. lutescens Zinovjev, 1990, О7 паратип; 43 - P. obsoleta Hennig, 1963, О7 голотип; $44-$ P. oxystomodes Emden, 1965, О7 паратип. 


\section{Phaonia chalchica grisea Zinovjev, nomen nudum}

MATERIAL. ZIN: Holotype, ơ: Карашокы бл. Бассага, Караганд. обл. вершина Грунин 17.V.957 Ph chalchica grisea subsp.n. Holotypus (= KAZAKHSTAN, Karaganda reg., Karashoky, $49.5^{\circ} \mathrm{N}$ $74.5^{\circ} \mathrm{E}, \mathrm{H} " 700 \mathrm{~m}$ asl, K. Grunin), 17.05.1957).

REMARKS. This specimen was marked by Zinovjev as the holotype of subsp. nov., but actually the new subspecies was never described him afterwards.

\section{Species described by A. Zinovjev \\ which type material is not in ZIN:}

Phaonia pusilla Zinovjev, 1990: 472

MATERIAL. One ZIN's 9 from Mongolia, Uvs prov., $3 \mathrm{~km} \mathrm{NE}$ somon Ondorkhangai, $49.29^{\circ} \mathrm{N} 94.89^{\circ} \mathrm{E}, 2200 \mathrm{~m}$ asl, Exp. Dr. Z. Kaszab, 21.06-16.07.1968 is marked as paratype by Zinovjev. But in the original description is indicated that "female unknown" [Zinovjev, 1990: 474], so this specimen could not be regarded as a paratype.

Other type material. MNM. Holotype $\sigma^{7}$, MONGOLIA, Tov (Central) prov., $66 \mathrm{~km} \mathrm{SEE} \mathrm{Ulan} \mathrm{Baator}\left(47.75^{\circ} \mathrm{N} 107.75^{\circ} \mathrm{E}\right)$, Exp. Dr. Z. Kaszab, 5.07.1963, (without mid legs). Paratypes, $3 \sigma^{7} \sigma^{7}$ MONGOLIA: Dornod prov., somon Khalkingol $\left(47.63^{\circ} \mathrm{N} 118.63^{\circ} \mathrm{E}\right)$, $600 \mathrm{~m}$ asl, Exp. Dr. Z. Kaszab, 12.08.1965, $10^{7}$; Uvs prov., $3 \mathrm{~km} \mathrm{NE}$ somon Ondorkhangai $\left(49.29^{\circ} \mathrm{N} 94.89^{\circ} \mathrm{E}\right), 2200 \mathrm{~m}$ asl, Exp. Dr. Z. Kaszab, 21.06-16.07.1968, $2 \sigma^{7} \sigma^{7}$ [Zinovjiev, 1990].

Phaonia aurata Zinovjev, 1992: 707

Phaonia bambusella Zinovjev, 1992: 700

Phaonia basisetosa Zinovjev, 1992: 709

Phaonia basisetosa subsp. trichaeta Zinovjev, 1992: 710

Phaonia himalaica Zinovjev, 1992: 704

In contrast to his previous habits, Zinovjev did not deposit parts of the type series of these species in ZIN collection, but returned all specimens to Natural History Museum of Denmark, University of Copenhagen, Denmark where they are presently deposited.

ACKNOWLEDGEMENTS. We thank Ludmila Kuznetsova and Galina Suleymanova (St-Petersburg) for their kind help in examination of ZIN's material. We thank Oleg Kosterin (Novosibirsk) and Maria Yanbulat (Moscow) for their advices and corrections.

\section{References}

Emden van F.I. 1965. The fauna of India and the adjacent countries. Diptera. Vol.7. Muscidae, Part 1. Delhi: Government of India. $647 \mathrm{pp}$.

Hennig W. 1963. Muscidae. [Part, Lieferung 233, 234 and 241.] // E. Lindner (Ed.) Die Fliegen der palaearktischen Region, 63b, Schweizerbart, Stuttgart. P.772-899.

Kozlov P.K. 1947. Монголия и Кам. Трехлетнее путешествие по Монголии и Тибету (1899-1901 г.г.) (= Mongolia and Kham. Three-year long journey to Mongolia and Tibet (1899-1901)) // $2^{\text {nd }}$ (reduced) Edition. OGIZ, Moscow. 437 pp. [In Russian].

Loew H. 1873. Diptera nova, in Pannonia inferiori et in confinibus Daciae regionibus a Ferd. Kowarzio capta // Berliner entomologische Zeitschrift . Vol.17. P.33-52.

Malyanov M.V. 1993. [Two new species of flies of the genus Phaonia R.-D. (Diptera, Muscidae) from North Tien Shan] // Entomologicheskoe Obozrenie. Vol.72. No.2. P.419-421 [in Russian].
Pont A.C. 1981. [A new species of the genus Phaonia (Diptera, Muscidae) from Siberia] // Entomologicheskoe Obozrenie. Vol.60. No.2. P. 427-429 [in Russian].

Pont A.C. 2013. The Fanniidae and Muscidae (Diptera) described by Paul Stein (1852-1921)// Zoosystematics and Evolution. Vol.89. No.1. P.31-166.

Pont A.C., Werner D. 2006. The Types of Fanniidae and Muscidae (Diptera) in the Museum für Naturkunde, Humboldt-Universität zu Berlin, Germany // Mitteilungen aus dem Museum für Naturkunde in Berlin Zoologische Reihe. Vol.82. No.1. P.3-139.

Roborovsky V.I. 1949. Путешествие в восточный Тянь-Шань и в Нань-Шань Труды экспедиции Русского географического общества по Центральной Азии в 1893-1895 гг. (= Journey to East Tian-Shan and Nian-Shan. Proceedings of the Expedition of the Russian Imperial Geographical Society to Central Asia executed in 1893-95). $2^{\text {nd }}$ (reduced) Edition. OGIZ, Moscow, 491 pp. [In Russian].

Rohlfien, K. \& Ewald B. 1974. Katalog der in den Sammlungen des ehemaligen Deutschen Entomologischen Institutes aufbewahrten Typen - XI. (Diptera: Cyclorrhapha: Schizophora: Calyptratae) // Beitrge zur Entomologie. Vol. 24. P.107-147.

Shinonaga S., Kano R. 1971. Fauna Japonica, Volume I, Muscidae (Insecta: Diptera) // Academic Press of Japan. 242 pp.

Sorokina V.S. 2015. New species of the genus Phaonia R.-D., 1830 (Diptera, Muscidae) from Central Asia // Zootaxa. Vol.4013. No.4. P. 571-587.

Stein P. 1907. Zur Kenntnis der Dipteren von Central-Asien. II. Cyclorrhapha schizophora schizometopa. Die von Roborowsky und Kozlov in der Mongolei und Tibet gesammelten Anthomyiiden // Ezhegodnik Zoologicheskogo Muzeya Imperatorskoy Akademii Nauk. Vol.12. P. 318-372.

Schnabl J. 1887. Contributions a la faune dipterologique. Genre Aricia // Trudy Russkogo Entomologicheskogo Obshchestva [Horae Societatis Entomologicae Rossicae]. Vol.22. P. 378-486.

Vikhrev N.E. 2012. Four new species of Lispe Latreille, 1796 (Diptera, Muscidae) with taxonomic notes on related species // Russian Entomological Journal. Vol. 21. No.4. P. 423-433.

Vikhrev N.E., Sorokina V.S. 2017. Taxanomic notes and faunistic data on the Muscidae (Diptera) of the Altai Mountains // Zootaxa [in press].

Zinovjev A.G. 1980a. [On the fauna of Phaonini (Diptera, Muscidae) of Mongolia, I] // Insects of Mongolia. Vol.7. P. 437-444 [in Russian].

Zinovjev A.G. 1980b. [Phaoniinae (Diptera, Muscidae) of the Far East] // Entomologicheskoe Obozrenie. Vol. 59. No.4. P.904913 [in Russian].

Zinovjev A.G. 1981. [Two new species of the genus Phaonia (Diptera, Muscidae) from the Soviet Far East] // Zoological Journal. Vol.60. No.4. P.623-625 [in Russian].

Zinovjev A.G. 1983. [Flies of the genus Phaonia R.-D. (Diptera, Muscidae) of the Middle Asia] // Entomologicheskoe Obozrenie. Vol.62. No.1. P.178-192 [in Russian].

Zinovjev A.G. 1987. [On the taxonomy of flies of the genus Phaonia R.-D. (Diptera, Muscidae)] // Entomologicheskoe Obozrenie. Vol.66. No.2. P.436-441 [in Russian].

Zinovjev A.G. 1990. [On the fauna of Phaoniini (Diptera, Muscidae) of Mongolia, II. The genera Lophosceles Ringdahl and Phaonia Robineau-Desvoidy] // Insects of Mongolia. Vol.11. P.471-514 [in Russian].

Zinovjev A.G. 1992. [On the fuana of flies of the genus Phaonia R.D. (Diptera, Muscidae) of West Himalayas] // Entomologicheskoe Obozrenie. Vol.71. No.3. P.700-712 [in Russian].

Zinovjev A.G. 1994. The genera Phaonia Robineau-Desvoidy and Lophosceles Ringdahl (Diptera, Muscidae) from the Caucasus Mountains // Dipterological Research. Vol.5. P.79-84. 